\title{
Central Limit Theorem for Leafwise Brownian Motions on Mapping Tori
}

\author{
Takehiko MORITA* and Kiyotaka SUZAKI \\ Osaka University \\ (Communicated by M. Kurihara)
}

\begin{abstract}
We consider the leafwise Brownian motion on a mapping torus of a homeomorphism on a compact metric space. We prove a central limit theorem for a class of additive functionals of the leafwise Brownian motion starting at any quasi-regular point and apply it to the leafwise Brownian motions on generalized Kronecker foliations. As an auxiliary result, we also give an elementary proof of the characterization of harmonic measures for the leafwise Brownian motion on the mapping torus.
\end{abstract}

\section{Introduction}

The notion of harmonic measures for leafwise Brownian motions on a compact foliated manifold was introduced by L. Garnett [8]. She showed that (1) such a measure always exists on any compact foliated Riemannian manifold, (2) any bounded Borel function on the manifold being harmonic along each leaf is constant on almost every leaf with respect to any finite harmonic measure, and (3) a Borel measure on the manifold is harmonic if and only if it is locally (in a given foliated chart) decomposed into a measure on the transversal and measures on the plaques each of which is expressed as a product of a positive harmonic function and the Riemannian volume of the plaque. After Garnett [8] and [9], characterization and statistical properties of harmonic measures are well studied by many authors in the case of Anosov foliations of negatively curved manifolds (e.g. [1], [10], [14], [16], [17], [21] etc.). A. Candel [2] improves Garnett's approach and extends it to the general theory of harmonic measures for foliated spaces. Candel considered a leafwise diffusion operator on a foliated space and defined the notion of harmonic measures with respect to it. We note that harmonic measures are characterized as invariant measures for the diffusion process generated by the operator. The basic results for foliated spaces and harmonic measures are available in [3] and [4].

Received September 11, 2013; revised November 3, 2014

Mathematics Subject Classification: 37C40 (Primary), 60J65, 60F05 (Secondary)

Key words and phrases: mapping torus, leafwise Brownian motion, harmonic measure, central limit theorem

*First author partially supported by the Grant-in-Aid for Scientific Research (B) 22340034, Japan Society for the Promotion of Science. 
In this paper we consider a mapping torus $M_{F}$ of a homeomorphism $F$ on a compact metric space $M$. It is one of the simplest foliated spaces, which is a topological space obtained by considering the product space $\mathbf{R} \times M$ and identifying $(u, x)$ with $(u-1, F x)$ for $u \in \mathbf{R}$ and $x \in M$. By the construction each leaf of $M_{F}$ inherits the usual flat structure from the real line. Therefore the leafwise Brownian motion $X=\left\{X^{z}\right\}_{z \in M_{F}}$ on $M_{F}$ is defined to be the diffusion process satisfying $X^{\pi_{F}(u, x)}(t)=\pi_{F}(u+B(t), x)$ for $t \geq 0$, where $\{B(t)\}_{t \geq 0}$ denotes a one-dimensional standard Brownian motion and $\pi_{F}: \mathbf{R} \times M \rightarrow M_{F}$ is the natural projection. For a continuous real valued, non constant function $f$ and $z \in M_{F}$, we consider the following stochastic process $A_{\lambda}^{z}=\left\{A_{\lambda}^{z}(t)\right\}$ with parameter $\lambda>0$ defined by

$$
A_{\lambda}^{z}(t)=\int_{0}^{\lambda t} f\left(X^{z}(s)\right) d s .
$$

We are interested in the central limit problem of the occupation time process $A_{\lambda}^{z}$ as $\lambda \rightarrow \infty$ under some appropriate conditions. We consider a point $z \in M_{F}$ for which there exists a harmonic probability measure $m_{z}$ such that $\lim _{t \rightarrow \infty}(1 / t) \int_{0}^{t} g\left(X^{z}(s)\right) d s=\int_{M_{F}} g d m_{z}$ holds almost surely for any continuous function $g$ on $M_{F}$. Such a point is called quasi-regular for $X$. The totality of quasi-regular points is denoted by $Q_{X}$. Note that it is not hard to see that each point in a closed leaf is quasi-regular. In addition, we show later that an arbitrary point of a leaf containing a quasi-regular point $z$ turns out to be quasi-regular and the corresponding harmonic measures are identical with $m_{z}$ (Proposition 2.4). The main purpose of this paper is to show the following.

THEOREM (Theorem 2.2 and Remark 2.4). Let $z$ be a quasi-regular point and $f$ a realvalued function which is not constant along each leaf. If there exists a leafwise $C^{2}$ function $g$ such that $f=\Delta_{L} g$, then the process $(1 / \sqrt{\lambda}) A_{\lambda}^{z}$ converges in law to the Brownian motion with variance $4 t \int_{M_{F}}\left(\nabla_{L} g\right)^{2} d m_{z}$ as $\lambda \rightarrow \infty$, where $\Delta_{L}$ and $\nabla_{L}$ denote the leafwise Laplacian and the leafwise gradient.

Next we make the following observation so as to explain about the significance of the limit theorem above. A leaf $L$ of $M_{F}$ is isometric to the one-dimensional torus $\mathbf{R} / n \mathbf{Z}$ with an integral perimeter $n$ or the real line $\mathbf{R}$ according as it is closed or not. In the former case, the leafwise Brownian motion restricted to $L$ behaves as the Brownian motion on $\mathbf{R} / n \mathbf{Z}$ and the normalized arc length measure $m$ on $L$ is a unique invariant measure for the restricted process to the leaf $L$. Thus it is not hard to verify that if $f$ satisfies $\int_{M_{F}} f d m=0$, then for any $z \in L$ the process $(1 / \sqrt{\lambda}) A_{\lambda}^{z}$ converges in law to a Brownian motion (not necessarily standard but with non-degenerate variance) as $\lambda \rightarrow \infty$. The invariant measure $m$ for the restricted process can be considered as an invariant measure for the original leafwise Brownian motion, that is, a harmonic measure supported on $L$. In the latter case, the leafwise Brownian motion restricted to the leaf $L$ behaves as the Brownian motion on $\mathbf{R}$. Since it is well-known that the Brownian motion on $\mathbf{R}$ is null recurrent and it does not have a finite invariant measure, it is not so easy to find an appropriate condition for the convergence in law of the process $(1 / \sqrt{\lambda}) A_{\lambda}^{z}$ starting 
at $z \in L$. For example, if $f$ is compactly supported on $L$, then for $z \in L$ either $(1 / \lambda)^{1 / 4} A_{\lambda}^{z}$ or $(1 / \sqrt{\lambda}) A_{\lambda}^{z}$ converges in law to a nontrivial process according as the integral of $f$ with respect to the Lebesgue measure on $L$ vanishes or not (see Theorem 4.4 in Section III-4.4 in [13]). But it often happens that a non closed leaf $L$ of $M_{F}$ is locally dense, that is, dense in a neighborhood of $L$ in $M_{F}$. In such a case, the restriction of $f$ to the leaf $L$ is neither compactly supported on $L$ nor integrable with respect to the Lebesgue measure on $L$. On the other hand, it is easy to see that if the leaf $L$ passes through a point which belongs to the $\pi_{F}$-image of the support of a non-atomic invariant measure for $F$, then $L$ is locally dense in $M_{F}$. Thus it turns out that we can not apply the above mentioned result on the Brownian motion on $\mathbf{R}$ to $L$ in many interesting cases.

Summing up the above argument, in the case when the starting point $z$ belongs to a closed leaf $L$, we have that there exists a natural harmonic probability measure and we can show the central limit theorem for $A_{\lambda}^{z}$ by the standard technique. On the other hand, in the case when the leaf $L$ containing the starting point $z$ is not closed, the leafwise Brownian motion restricted to $L$ does not have a finite invariant measure. In particular, if the underlying dynamical system $F$ has non-atomic invariant measures, the global continuity of the observable $f$ prevents us from applying the known limit theorem for one-dimensional Brownian motion to the restricted process. Therefore, the existence of non-atomic invariant probability measure of $F$ seems to make the problem complicated at the first glance. But our result illustrates that it is not so as it seems. In fact, there is a one-to-one correspondence between the set of harmonic probability measures for the leafwise Brownian motion $X$ on the mapping torus $M_{F}$ and that of invariant probability measures for the underlying dynamical system $F$. The correspondence is natural and explicit in the sense that if a harmonic measure $m$ for $X$ is corresponding to an invariant measure $\mu$ of $F$, we have

$$
\int_{M_{F}} f(z) d m=\int_{[0,1) \times M}\left(f \circ \pi_{F}\right)(u, x) d(l \times \mu)
$$

for any continuous function $f$ on $M_{F}$, where $l$ is the Lebesgue measure on the unit interval $[0,1)$. We can investigate the asymptotic behavior of the leafwise Brownian motion with respect to harmonic measures by using the ergodic properties of the invariant measures for underlying dynamical system. Thus the correspondence and its auxiliary results play important roles in this paper. Noting that there are no non-constant nonnegative harmonic functions on $\mathbf{R}$, we see that the correspondence above is deduced from the local characterization of harmonic measures in the general theory (e.g. see [4] and [14]). But we shall treat it as Theorem 2.1 and give it an elementary proof in the sake of self-containedness.

The paper is organized as follows. In Section 2, we give the statement of our results after reviewing some fundamental facts. Section 3 is devoted to the proof of Theorem 2.1. We prove the main theorem (Theorem 2.2) in Section 4. Finally we apply our results to the case when the underlying homeomorphism is uniquely ergodic in Section 5. We note that we try to make our arguments as self-contained as possible except for the use of the basic facts in stochastic analysis and the infinitesimal generator for contraction semi-group. We note that 
the basic notions and results that we need can be found in Chapter I, II, and III in [13] and Chapter VIII in [6]. So we do not need the general theory of foliated space and harmonic measures established in [2] and [4].

\section{Preliminaries and the results}

First of all we recall some notions. Let $M$ be a compact metric space and let $F$ be a homeomorphism of $M$ onto itself. Obviously, the product space $\mathbf{R} \times M$ is a foliated space with leaves $\mathbf{R} \times\{x\}$ for each $x \in M$. The continuous $\mathbf{Z}$-action defined on $\mathbf{R} \times M$ by $\mathbf{Z} \times(\mathbf{R} \times M) \ni$ $(k, u, x) \mapsto\left(u-k, F^{k} x\right) \in \mathbf{R} \times M$ is properly discontinuous and it maps leaves to leaves. The quotient space $(\mathbf{R} \times M) / \mathbf{Z}$ is a compact foliated space called the mapping torus of $F$ and we denote it by $M_{F}$. For $(u, x) \in \mathbf{R} \times M$, we often write as $[u, x]=\pi_{F}(u, x)$ to denote the equivalence class containing $(u, x)$, where $\pi_{F}: \mathbf{R} \times M \rightarrow M_{F}$ is the natural projection. Clearly $\pi_{F}$ restricted to $(u-1 / 2, u+1 / 2) \times M$ is a homeomorphism onto its image $U_{u}=\pi_{F}((u-1 / 2, u+1 / 2) \times M)$ for each $u \in \mathbf{R}$. In particular, $\left(U_{u}, \varphi_{u}\right)$ gives a foliated chart for $M_{F}$, where $\varphi_{u}$ is the inverse branch of $\pi_{F}$ on $U_{u}$. Let $C\left(M_{F}\right)$ be the Banach space of continuous functions on $M_{F}$ endowed with the supremum norm $\|\cdot\|_{\infty}$. One can easily see that a function $f$ on $M_{F}$ belongs to $C\left(M_{F}\right)$ if and only if $f \circ \pi_{F}$ is a continuous function on $\mathbf{R} \times M$. Furthermore, $f \in C\left(M_{F}\right)$ implies that the family $\left\{f \circ \pi_{F}(u, x)\right\}_{x \in M}$ of functions in $u \in \mathbf{R}$ is equicontinuous and each element of which is uniformly continuous since both $M$ and $M_{F}$ are compact. Namely we have

$$
\begin{array}{ll}
\lim _{\delta \downarrow 0} \sup _{v:|v-u|<\delta} \sup _{x \in M}\left|f \circ \pi_{F}(v, x)-f \circ \pi_{F}(u, x)\right|=0 & \text { for each } u \in \mathbf{R} \text { and } \\
\lim _{\delta \downarrow 0} \sup _{u, v \in \mathbf{R}:|u-v|<\delta}\left|f \circ \pi_{F}(u, x)-f \circ \pi_{F}(v, x)\right|=0 & \text { for each } x \in M .
\end{array}
$$

The leaf of $M_{F}$ passing through $z \in M_{F}$ is denoted by $L_{z}$. It is easy to see that $L_{[u, x]}$ is identified with a one-dimensional flat torus with perimeter $n$ if $x$ is a periodic point of $F$ with the least period $n$ and $L_{[u, x]}$ is identified with $\mathbf{R}$ if $x$ is not periodic point of $F$. In any case leaves are one-dimensional smooth manifold with the usual flat metric and we can consider differentiation along the leaf. Therefore the gradient $\nabla_{L} f$ along the leaf can be defined so that the formula

$$
\nabla_{L} f([u, x])=\frac{\partial\left(f \circ \pi_{F}\right)}{\partial u}(u, x)
$$

holds if $f$ is of class $C^{1}$ along each leaf. For nonnegative integer $r$ let $C_{L}^{r}\left(M_{F}\right)$ be the totality of functions $f$ of class $C^{r}$ along each leaf satisfying $\left(\nabla_{L}\right)^{k} f \in C\left(M_{F}\right)$ for any $k$ with $0 \leq k \leq r$. Since it is easy to construct a function in $C_{L}^{\infty}\left(M_{F}\right)$ separating given two points in $M_{F}$, the Stone-Weierstrass theorem yields that $C_{L}^{\infty}\left(M_{F}\right)$ is dense in $C\left(M_{F}\right)$. One can easily see that a function $f$ on $M_{F}$ belongs to $C_{L}^{r}\left(M_{F}\right)$ if and only if $f \circ \pi_{F}$ is $C_{L}^{r}(\mathbf{R} \times M)$ i.e. $f \circ \pi_{F}$ is $r$ times partially differentiable function on $\mathbf{R} \times M$ in the first coordinate and its 
partial derivative $\left(\nabla_{L}\right)^{k} f \circ \pi_{F}=\partial^{k}\left(f \circ \pi_{F}\right) / \partial u^{k}$ is a continuous function on $\mathbf{R} \times M$ for each $k$ with $0 \leq k \leq r$. Clearly the leafwise Laplacian $\Delta_{L}$ is given by the formula

$$
\Delta_{L} f([u, x])=\left(\nabla_{L}\right)^{2} f([u, x])=\frac{\partial^{2}\left(f \circ \pi_{F}\right)}{\partial u^{2}}(u, x)
$$

for $f \in C_{L}^{2}\left(M_{F}\right)$.

The following is an easy consequence of the fundamental theorem of calculus.

Proposition 2.1. Consider a function $f \in C\left(M_{F}\right)$. For the existence of a solution $g \in C_{L}^{1}\left(M_{F}\right)$ of the equation $\nabla_{L} g=f$, it is necessary and sufficient that there exists a function $\varphi \in C(M)$ satisfying

$$
\int_{0}^{1} f([u, x]) d u=\varphi(F x)-\varphi(x)
$$

for any $x \in M$. Moreover, for the existence of the solution $h \in C_{L}^{2}\left(M_{F}\right)$ of the equation $\Delta_{L} h=f$, it is necessary and sufficient that there exist $\varphi$ and $\psi \in C(M)$ satisfying the equations (4) and

$$
\int_{0}^{1} u f([u, x]) d u=\varphi(F x)-(\psi(F x)-\psi(x))
$$

for any $x \in M$.

PROOF. First assume that $g \in C_{L}^{1}\left(M_{F}\right)$ satisfies $\nabla_{L} g=f$. By virtue of the fundamental theorem of calculus, we have

$$
g([t, x])=g([0, x])+\int_{0}^{t} f([u, x]) d u
$$

holds for $(t, x) \in \mathbf{R} \times M$. Since $f([u-1, F x])=f([u, x])$ and $g([u-1, F x])=g([u, x])$ hold for $(u, x) \in \mathbf{R} \times M$, we have

$$
\begin{aligned}
g([t-1, F x]) & =g([0, F x)]+\int_{0}^{t-1} f([u, F x]) d u \\
& =g([0, F x])+\int_{0}^{t-1} f([u+1, x]) d u \\
& =g([0, F x])+\int_{1}^{t} f([u, x]) d u \\
& =g([0, F x])-g([0, x])-\int_{0}^{1} f([u, x]) d u+g([t, x])=g([t, x]) .
\end{aligned}
$$

Thus we reach the equation (4) with $\varphi(x)=g([0, x])$. 
Conversely, assume that there exists $\varphi \in C(M)$ satisfying the equation (4) for any $x \in$ $M$. Define $\tilde{g}: \mathbf{R} \times M \rightarrow \mathbf{C}$ by

$$
\tilde{g}(t, x)=\varphi(x)+\int_{0}^{t} f([u, x]) d u .
$$

Then by using the identity $f([u-1, F x])=f([u, x])$ again, we have

$$
\begin{aligned}
\tilde{g}(t-1, F x) & =\varphi(F x)+\int_{0}^{t-1} f([u, F x]) d u \\
& =\varphi(F x)+\int_{0}^{t-1} f([u+1, x]) d u \\
& =\varphi(F x)+\int_{1}^{t} f([u, x]) d u .
\end{aligned}
$$

It is easy to see from the equation (4) that the function in the last line equals $\tilde{g}(t, x)$. Thus $\tilde{g}$ determines the function $g \in C_{L}^{1}\left(M_{F}\right)$. Now the proof of the first assertion is complete.

In order to prove the second assertion, we may assume that there exists $g \in C_{L}^{1}\left(M_{F}\right)$ such that it satisfies $\nabla_{L} g=f$ and the equation (4) holds for $x \in M$ with $\varphi(x)=g([0, x])$. It suffices to show that the equation $\nabla_{L} h=g$ have a solution in $C_{L}^{1}\left(M_{F}\right)$ if and only if there exists $\psi \in C(M)$ satisfying the equation (5) for any $x \in M$. On the other hand we see from the first assertion that the former is equivalent to the existence of $\psi \in C(M)$ such that

$$
\int_{0}^{1} g([t, x]) d t=\psi(F x)-\psi(x)
$$

for any $x \in M$. Since we know that $g([t, x])=\int_{0}^{t} f([u, x]) d u+\varphi(x)$ for $(t, x) \in \mathbf{R} \times M$, the left hand side of the above equation is calculated as

$$
\begin{aligned}
\int_{0}^{1} g([t, x]) d t & =\int_{0}^{1}\left(\int_{0}^{t} f([u, x]) d u\right) d t+\varphi(x) \\
& =\int_{0}^{1}(1-u) f([u, x]) d u+\varphi(x) \\
& =\varphi(F x)-\int_{0}^{1} u f[(u, x]) d u
\end{aligned}
$$

by the Fubini theorem and the equation (4). Thus we arrive at the desired result.

Next we introduce leafwise Brownian motions on the mapping torus. Let $B=\{B(t)\}_{t \geq 0}$ be a one-dimensional Brownian motion starting at 0 defined on a probability space $(\Omega, \mathcal{F}, P)$. Given $(u, x) \in \mathbf{R} \times M$ the leafwise Brownian motion on $M_{F}$ starting at $[u, x]$ is an $M_{F}$-valued stochastic process $X^{[u, x]}=\left\{X^{[u, x]}(t)\right\}_{t \geq 0}$ defined by $X^{[u, x]}(t)=[u+B(t), x]$ for $t \geq 0$. 
$X^{[u, x]}=\left\{X^{[u, x]}(t)\right\}_{t \geq 0}$ is a diffusion process on the mapping torus $M_{F}$ as well as a diffusion process on the leaf $L_{[u, x]}$. In fact, we can show that $X^{[u, x]}=\left\{X^{[u, x]}(t)\right\}_{t \geq 0}$ gives a Feller semi-group on $C\left(M_{F}\right)$. To this end and for later use for $t \geq 0$ and a bounded Borel function $f$ on $M_{F}$, we define

$$
T(t) f([u, x])=E\left[f\left(X^{[u, x]}(t)\right)\right]=E[f([u+B(t), x])] .
$$

We note that we can rewrite it as

$$
\begin{aligned}
T(t) f([u, x]) & =\sum_{k \in \mathbf{Z}} E[f([u+B(t), x]) ; k \leq u+B(t)<k+1] \\
& =\sum_{k \in \mathbf{Z}} \int_{[k, k+1)} f([v, x]) p(t, v-u) d v \\
& =\sum_{k \in \mathbf{Z}} \int_{[0,1)} f\left(\left[v, F^{k} x\right]\right) p(t, v+k-u) d v,
\end{aligned}
$$

where $p(t, v)=(1 / \sqrt{2 \pi t}) \exp \left(-v^{2} / 2 t\right)$. In the case of the mapping torus it is not hard to prove the following. We give a proof for our convenience.

Proposition 2.2. The family of positive operators $\{T(t)\}_{t \geq 0}$ restricted to $C\left(M_{F}\right)$ turns out to be a Feller semi-group. Precisely we have the following.

(1) For any $t \geq 0$ we have $T(t) C\left(M_{F}\right) \subset C\left(M_{F}\right)$.

(2) For $s, t \geq 0$ and $f \in C\left(M_{F}\right)$, we have $T(s+t) f=T(t) T(s) f$.

(3) For any $t \geq 0$ and $f \in C\left(M_{F}\right),\|T(t) f\|_{\infty} \leq\|f\|_{\infty}$.

(4) For any $f \in C\left(M_{F}\right), \lim _{t \downarrow 0}\|T(t) f-f\|_{\infty}=0$.

(5) The domain $\mathcal{D}(A)$ of the infinitesimal generator $A$ of the semi-group contains the space $C_{L}^{2}\left(M_{F}\right)$ and $f \in C_{L}^{2}\left(M_{F}\right)$ yields $A f=(1 / 2) \Delta_{L} f$.

Proof. First we recall that $f \in C\left(M_{F}\right)$ if and only if $f \circ \pi_{F} \in C(\mathbf{R} \times M)$. By the definition (6), the assertion (1) follows from the bounded convergence theorem. The assertion (2) follows immediately from the Markov property of the Brownian motion $B=\{B(t)\}_{t \geq 0}$, since we see that for fixed $x \in M f([u, x])=f \circ \pi_{F}(u, x)$ is a bounded continuous function in $u \in \mathbf{R}$. The assertion (3) is obvious from the definition (6). The assertion (4) is verified as follows. Take any $f \in C\left(M_{F}\right)$. Recall that the family $\left\{f \circ \pi_{F}(u, x)\right\}_{x \in M}$ of functions in $u$ is equicontinuous and the first equation in (1) holds. Therefore we see that for any $\varepsilon>0$, there exists $\delta>0$ such that $\sup _{x \in M, u, v \in \mathbf{R}:|u-v|<\delta}|f([u, x])-f([v, x])|<\varepsilon$. Thus we have for any $(u, x) \in \mathbf{R} \times M$

$$
\begin{aligned}
&|T(t) f([u, x])-f([u, x])|=|E[f([u+B(t), x])-f([u, x])]| \\
& \leq E[\midf([u+B(t), x])-f([u, x])|;| B(t) \mid<\delta] \\
& \quad+E[|f([u+B(t), x])-f([u, x])| ;|B(t)| \geq \delta] \\
&<\varepsilon+2\|f\|_{\infty} P(|B(t)| \geq \delta) \rightarrow 0 \quad(t \rightarrow 0) .
\end{aligned}
$$


This implies that the assertion (4) is valid.

It remains to prove the assertion (5). For $f \in C_{L}^{2}\left(M_{F}\right)$ we have only to show that

$$
\lim _{t \rightarrow 0}\left\|\frac{T(t) f-f}{t}-\frac{1}{2} \Delta_{L} f\right\|_{\infty}=0 .
$$

Since for fixed $x \in M f \circ \pi_{F}(u, x)$ is $C^{2}$ function in $u$, we can apply the Itô formula to $f \circ \pi_{F}$. Thus we have

$f([u+B(t), x])-f([u, x])=\int_{0}^{t} \nabla_{L} f([u+B(s), x]) d B(s)+\frac{1}{2} \int_{0}^{t} \Delta_{L} f([u+B(s), x]) d s$.

Since the first term in the right hand side is a martingale with mean zero, by taking expectation we have

$$
T(t) f([u, x])-f([u, x])=\int_{0}^{t} \frac{1}{2} T(s) \Delta_{L} f([u, x]) d s .
$$

Therefore we have

$$
\frac{T(t) f([u, x])-f([u, x])}{t}-\frac{1}{2} \Delta_{L} f([u, x])=\frac{1}{2 t} \int_{0}^{t}\left(T(s) \Delta_{L} f([u, x])-\Delta_{L} f([u, x])\right) d s .
$$

Applying the assertion (4) to $\Delta_{L} f$, we obtain the desired result.

REMARK 2.1. The corresponding result to Proposition 2.2 plays a crucial role in the general theory of leafwise diffusions on foliated spaces in [2] but it is not so easy as in the present case.

In what follows we call the stochastic process $X^{z}=\left\{X^{z}\right\}_{t \geq 0}$ defined in the above the leafwise Brownian motion on $M_{F}$ starting at $z$. The family $X=\left\{X^{z}\right\}_{z \in M_{F}}$ of the leafwise Brownian motions is simply called the leafwise Brownian motion on $M_{F}$.

Now we consider harmonic measures. A Borel measure $m$ on $M_{F}$ is called a harmonic measure for the leafwise Brownian motion if

$$
\int_{M_{F}} \Delta_{L} f(z) d m=0
$$

holds for any $f \in C_{L}^{2}\left(M_{F}\right)$. Combining Proposition 2.2 with the fact that $C_{L}^{2}\left(M_{F}\right)$ is dense in $C\left(M_{F}\right)$, we easily show that the harmonic measure is characterized as an invariant measure for the leafwise Brownian motion.

PROPOSITION 2.3. A Borel probability measure $m$ on $M_{F}$ is a harmonic measure for leafwise Brownian motion if and only if

$$
\int_{M_{F}} T(t) f(z) d m=\int_{M_{F}} f(z) d m
$$

holds for any $f \in C\left(M_{F}\right)$ and for any $t \geq 0$. 
Proof. Assume that a Borel probability measure $m$ satisfies (9) for any $f \in C\left(M_{F}\right)$ and for any $t \geq 0$. Consider the case when $f \in C_{L}^{2}\left(M_{F}\right)$. The assertion (5) in Proposition 2.2 guarantees us to differentiate the constant function $t \mapsto \int_{M_{F}} T(t) f(z) d m$ by differentiating the integrand. Thus we obtain the equation (8). Conversely, assume that a Borel probability measure $m$ is harmonic. Since $C_{L}^{2}\left(M_{F}\right)$ is dense in $C\left(M_{F}\right)$, it suffice to show the equation (9) for any $f \in C_{L}^{2}\left(M_{F}\right)$. If we notice the fact that $T(t) f \in C_{L}^{2}\left(M_{F}\right)$ for $t \geq 0$ and the assertion (5) in Proposition 2.2 yields

$$
\lim _{h \rightarrow 0}\left\|\frac{T(t+h) f-T(t) f}{h}-\frac{1}{2} \Delta_{L} T(t) f\right\|_{\infty}=0,
$$

we easily see that the function $t \mapsto \int_{M_{F}} T(t) f(z) d m$ is differentiable with derivative $\int_{M_{F}}(1 / 2) \Delta_{L} T(t) f(z) d m=0$. Thus we reach the equality (9).

REMARK 2.2. By virtue of Proposition 2.3, we see that the strongly continuous contraction semi-group $\{T(t)\}_{t \geq 0}$ on $C\left(M_{F}\right)$ extends to a strongly continuous contraction semigroup on $L^{p}(m)$ with $1 \leq p<\infty$ for any harmonic probability measure $m$. We also denote it by $\{T(t)\}_{t \geq 0}$.

Now we state the structure theorem of harmonic measures for leafwise Brownian motion. Since it plays an important role in this paper, we give an elementary proof and some auxiliary results in the next section.

THEOREM 2.1. Let $\mu$ be an invariant measure for a homeomorphism $F$ from a compact metric space $M$ onto itself. Consider the measure $m_{\mu}$ defined by

$$
\int_{M_{F}} f(z) d m_{\mu}=\int_{[0,1) \times M} f([u, x]) d(l \times \mu)
$$

for each continuous function $f$ on $M_{F}$, where $l$ is the one-dimensional Lebesgue measure. Then $m_{\mu}$ is a harmonic measure for the leafwise Brownian motion on $M_{F}$. Conversely, if $m$ is any harmonic measure for the leafwise Brownian motion on $M_{F}$, there exists a unique invariant measure $\mu$ for $F$ such that $m$ is given as $m_{\mu}$ defined by the equality (10).

REMARK 2.3. By virtue of Theorem 2.1, there exists a natural one to one correspondence between the set of invariant probability measures $\mathcal{I M}(F)$ for the homeomorphism $F$ and that of harmonic probability measures $\mathcal{H M}(X)$ for the leafwise Brownian motion. Therefore we can say that the study of the members of $\mathcal{H} \mathcal{M}(X)$ is equivalent to that of the members of $\mathcal{I} \mathcal{M}(F)$. At least in this sense, the ergodic theory of a dynamical system via invariant measures is regarded as a special case of the ergodic theory of a foliated space via harmonic measures.

In order to state the main results we need the notion of quasi-regular point for the leafwise Brownian motion. To this end we first recall the notion of quasi-regular point of a dynamical system (e.g. see [5]). For a homeomorphism $F$ on a compact metric space, a point $x \in M$ is 
called a quasi-regular point for $F$ if for any $f \in C(M)$ the $\operatorname{limit}_{n \rightarrow \infty}(1 / n) \sum_{k=0}^{n-1} f\left(F^{k} x\right)$ exists. The totality of quasi-regular points for $F$ is denoted by $Q_{F}$. Following the definition of quasi-regular points for a homeomorphism, we call a point $z \in M_{F}$ a quasi-regular point for the leafwise Brownian motion $X$ if for any $f \in C\left(M_{F}\right)$ the $\operatorname{limit}_{t \rightarrow \infty}(1 / t) \int_{0}^{t} f\left(X^{z}(s)\right) d s$ exists $P$-a.s. The totality of quasi-regular points for $X$ is denoted by $Q_{X}$. Note that the set $Q_{X}$ is depending only on the law of the Brownian motion $B$ by definition. Indeed, if we choose another Brownian motion $B^{\prime}$ and consider the corresponding leafwise Brownian motion $X^{\prime}$, we see that the laws of $X$ and $X^{\prime}$ coincide and consequently we have $Q_{X}=Q_{X^{\prime}}$. We summarize the basic facts concerned with the quasi-regular points for $X$ in the following proposition.

Proposition 2.4. (1) Consider the set

$$
\begin{aligned}
& Q_{X}\left(M_{F} \times \Omega\right)=\left\{(z, \omega) \in M_{F} \times \Omega\right. \\
&\left.: \lim _{t \rightarrow \infty}(1 / t) \int_{0}^{t} f\left(X^{z}(s, \omega)\right) d s \text { exists for any } f \in C\left(M_{F}\right)\right\},
\end{aligned}
$$

then $Q_{X}=\left\{z \in M_{F}: P\left(Q_{X}\left(M_{F} \times \Omega\right)_{z}\right)=1\right\}$. Consequently, $Q_{X}$ is Borel measurable subset of $M_{F}$.

(2) $m\left(Q_{X}\right)=1$ for any harmonic probability measure $m$ for $X$.

(3) For any $z \in Q_{X}$, we have $L_{z} \subset Q_{X}$. In particular, there exist a harmonic probability measure $m_{z}$ for $X$ and a measurable set $\Lambda$ with $P(\Lambda)=1$ such that $\omega \in \Lambda$ yields

$$
\lim _{t \rightarrow \infty} \frac{1}{t} \int_{0}^{t} f\left(X^{w}(s, \omega)\right) d s=\int_{M_{F}} f d m_{z}
$$

for any $w \in L_{z}$ and $f \in C\left(M_{F}\right)$.

(4) For any $z \in Q_{X}, m_{z}$ is a unique measure such that the equality (11) holds for $w=z$ and for any $f \in C\left(M_{F}\right)$. Consequently, we have $m_{w}=m_{z}$ for all $w \in L_{z}\left(\subset Q_{X}\right)$.

PROOF.

(1) Obviously the set $Q_{X}(f)=\left\{(z, \omega) \in M_{F} \times \Omega: \lim _{t \rightarrow \infty}(1 / t) \int_{0}^{t} f\left(X^{z}(s, \omega)\right) d s\right.$ exists $\}$ is $\mathcal{B}\left(M_{F}\right) \times \mathcal{F}$-measurable for each $f \in C\left(M_{F}\right)$. Since $C\left(M_{F}\right)$ is a separable Banach space, we can choose a countable subset $\left\{f_{n}\right\}$ which is dense in $C\left(M_{F}\right)$. It is easy to see that $Q_{X}\left(M_{F} \times \Omega\right)=\bigcap_{n=1}^{\infty} Q_{X}\left(f_{n}\right)$. Therefore $Q_{X}\left(M_{F} \times \Omega\right)$ is $\mathcal{B}\left(M_{F}\right) \times \mathcal{F}$-measurable. Thus the Fubini theorem for measurable sets yields that the $z$-section $Q_{X}\left(M_{F} \times \Omega\right)_{z}$ is $\mathcal{F}$-measurable for each $z \in M_{F}$ and the function $\varphi(z)=P\left(Q_{X}\left(M_{F} \times \Omega\right)_{z}\right)$ is $\mathcal{B}\left(M_{F}\right)$ measurable. Hence $Q_{X}=\varphi^{-1}(\{1\})$ is $\mathcal{B}\left(M_{F}\right)$-measurable.

(2) Since the set $Q_{X}$ depends only on the law of the Brownian motion, we may assume that $(\Omega, \mathcal{F}, P)$ is the canonical one-dimensional Wiener space. i.e. $\Omega=C([0, \infty) \rightarrow \mathbf{R})$ is the totality of continuous real-valued functions $\omega$ on $[0, \infty)$ endowed with the metric $\rho\left(\omega_{1}, \omega_{2}\right)=\sum_{n=1}^{\infty} 2^{-n}\left(\left(\max _{0 \leq t \leq n}\left|\omega_{1}(t)-\omega_{2}(t)\right|\right) \wedge 1\right)$ for $\omega_{1}, \omega_{2} \in \Omega, \mathcal{F}$ is the topological 
Borel field of $\Omega$, and $P=P_{0}$ is the one-dimensional Wiener measure with the initial distribution $\delta_{0}$ (see Section I-4 and Section I-7 in [13]). Note that the Brownian motion $B=\{B(t)\}$ starting at 0 is given by $B(\omega, t)=\omega(t)$ in this case. Therefore the leafwise Brownian motion $X^{[u, x]}$ starting at $[u, x]$ with $(u, x) \in \mathbf{R} \times M$ is given by $X^{[u, x]}(t, \omega)=[u+\omega(t), x]$. We consider the semi-flow of translations $\left\{\theta_{t}\right\}_{t \geq 0}$ on $\Omega$ defined by

$$
\left(\theta_{t} \omega\right)(s)=\omega(t+s)-\omega(t) \text { for } s \geq 0 .
$$

The translation invariance of the Wiener measure implies that $\left(\left\{\theta_{t}\right\}, P\right)$ is a continuous parameter measure-preserving dynamical system. We define a semi-flow $\left\{\Theta_{t}\right\}_{t \geq 0}$ of skew product transformations on $M_{F} \times \Omega$ by

$$
\Theta_{t}(z, \omega)=\left(X^{z}(t, \omega), \theta_{t} \omega\right)
$$

for $(z, \omega) \in M_{F} \times \Omega$. The semi-group property of $\left\{\Theta_{t}\right\}_{t \geq 0}$ is verified as follows. Write as $z=[u, x]$ with $(u, x) \in \mathbf{R} \times M$. Then we have

$$
\begin{aligned}
\Theta_{t+s}([u, x], \omega) & =\left([u+\omega(t+s), x], \theta_{t+s} \omega\right)=\left(\left[u+\omega(s)+\left(\theta_{s} \omega\right)(t), x\right], \theta_{t}\left(\theta_{s} \omega\right)\right) \\
& =\Theta_{t}\left([u+\omega(s), x], \theta_{s} \omega\right)=\Theta_{t}\left(\Theta_{s}([u, x], \omega)\right) .
\end{aligned}
$$

For a Borel probability measure $m$ on $M_{F}$, it is easy to show that $m$ is a harmonic measure for $X$ if and only if $m \times P$ is an invariant measure for $\left\{\Theta_{t}\right\}$. Therefore if $m$ is a harmonic probability measure for $X$, we can apply the ergodic theorem to the continuous parameter measure-preserving dynamical system $\left(\left\{\Theta_{t}\right\}, m \times P\right)$ and the function $g$ on $M_{F} \times \Omega$ expressed as $g(z, \omega)=f(z)$ for $(z, \omega) \in M_{F} \times \Omega$, where $f \in C\left(M_{F}\right)$. Noticing the equation $g\left(\Theta_{t}(z, \omega)\right)=f\left(X^{z}(t, \omega)\right)$, we see that $\lim _{t \rightarrow \infty}(1 / t) \int_{0}^{t} f\left(X^{z}(s, \omega)\right) d s$ exists $m \times$ $P$-a.e. $(z, \omega)$. Therefore we have $(m \times P)\left(Q_{X}(f)\right)=1$ and consequently, we have $(m \times$ $P)\left(Q_{X}\left(M_{F} \times \Omega\right)\right)=1$. Thus $P\left(Q_{X}\left(M_{F} \times \Omega\right)_{z}\right)=1$ holds $m$-a.e.z. This yields $m\left(Q_{X}\right)=1$.

(3) We may assume that $(\Omega, \mathcal{F}, P)$ is the canonical one-dimensional Wiener space again. We first recall the well-known fact that if $T$ is a tail event, then we have $P_{u}(T)=1$ for all $u \in \mathbf{R}$ or $P_{u}(T)=0$ for all $u \in \mathbf{R}$, where $P_{u}$ is the probability measure on $(\Omega, \mathcal{F})$ so that $\omega=\{\omega(t)\}_{t \geq 0}$ is observed as the Brownian sample path starting at $u P_{u}$-a.s. i.e. the measure defined by $P_{u}(A)=P(\{\omega: \omega+u \in A\})$ for $A \in \mathcal{F}$ in our case. We note that a detailed proof of the fact can be found in p.17 of [7] for example.

For $f \in C\left(M_{F}\right), c \in \mathbf{R}, v \in \mathbf{R}$, and $x \in M$, the set $\Lambda(f, c, v, x)=\{\omega \in \Omega$ : $\left.\lim _{t \rightarrow \infty}(1 / t) \int_{0}^{t} f([v+\omega(s), x]) d s=c\right\}$ is obviously a tail event. By the definition of quasiregular point we see that if $z=[u, x]$ is a quasi-regular point, there exists a constant $c(f, x)$ depending only on $x$ and $f \in C\left(M_{F}\right)$ such that

$$
\begin{aligned}
& P(\Lambda(f, c(f, x), v, x))=P_{v}(\Lambda(f, c(f, x), 0, x))=P_{u}(\Lambda(f, c(f, x), 0, x)) \\
& \quad=P(\Lambda(f, c(f, x), u, x))=1 .
\end{aligned}
$$

Choose a countable dense set $\left\{f_{n}\right\}$ in $C\left(M_{F}\right)$ and put $\Lambda=\bigcap_{n=1}^{\infty} \bigcap_{v \in \mathbf{Q}} \Lambda\left(f_{n}, c\left(f_{n}, x\right), v, x\right)$. Then obviously we have $P(\Lambda)=1$ and we can show that $\Lambda$ is the desired set in the assertion 
(3) as follows. For any $f \in C\left(M_{F}\right)$ and $\varepsilon>0$, there exists $n$ such that $\left\|f-f_{n}\right\|_{\infty}<\varepsilon$. Clearly we see that $\left|c(f, x)-c\left(f_{n}, x\right)\right|<\varepsilon$. Therefore we have for any $v \in \mathbf{Q}$ and $\omega \in \Lambda$

$$
\begin{aligned}
\limsup _{t \rightarrow \infty}\left|\frac{1}{t} \int_{0}^{t} f\left(X^{[v, x]}(s, \omega)\right) d s-c(f, x)\right| \\
\leq \limsup _{t \rightarrow \infty}\left|\frac{1}{t} \int_{0}^{t} f\left(X^{[v, x]}(s, \omega)\right) d s-\frac{1}{t} \int_{0}^{t} f_{n}\left(X^{[v, x]}(s, \omega)\right) d s\right| \\
+\lim _{t \rightarrow \infty}\left|\frac{1}{t} \int_{0}^{t} f_{n}\left(X^{[v, x]}(s, \omega)\right) d s-c\left(f_{n}, x\right)\right|+\left|c\left(f_{n}, x\right)-c(f, x)\right|<2 \varepsilon .
\end{aligned}
$$

Next choose any $u \in \mathbf{R}$ and $\varepsilon>0$. Then the second assertion in (1) yields that there exists $\delta>0$ such that $\left|f\left(X^{[u, x]}(t, \omega)\right)-f\left(X^{[v, x]}(t, \omega)\right)\right|=|f([u+\omega(t), x])-f([v+\omega(t), x])|<\varepsilon$ for any $v \in \mathbf{R}$ with $|u-v|<\delta$. Therefore if we choose $v \in \mathbf{Q}$ with $|u-v|<\delta$, we have

$$
\begin{array}{r}
\limsup _{t \rightarrow \infty}\left|\frac{1}{t} \int_{0}^{t} f\left(X^{[u, x]}(s, \omega)\right) d s-c(f, x)\right| \\
\leq \limsup _{t \rightarrow \infty}\left|\frac{1}{t} \int_{0}^{t} f\left(X^{[u, x]}(s, \omega)\right) d s-\frac{1}{t} \int_{0}^{t} f\left(X^{[v, x]}(s, \omega)\right) d s\right| \\
\quad+\lim _{t \rightarrow \infty}\left|\frac{1}{t} \int_{0}^{t} f\left(X^{[v, x]}(s, \omega)\right) d s-c(f, x)\right| \leq \varepsilon .
\end{array}
$$

Hence we have $\lim _{t \rightarrow \infty}(1 / t) \int_{0}^{t} f\left(X^{[v, x]}(s, \omega)\right) d s=c(f, x)$ holds for any $f \in C\left(M_{F}\right)$, $v \in \mathbf{R}$, and $\omega \in \Lambda$. Finally, since $c(\cdot, x): C\left(M_{F}\right) \rightarrow \mathbf{C}$ is bounded positive linear functional with $c(1, x)=1$, there exists a probability measure $m_{z}$ satisfying $c(f, x)=\int_{M_{F}} f d m_{z}$ by the Riesz representation theorem. Obviously, $m_{z}$ is a harmonic measure for $X$.

(4) It follows immediately from the assertion (3).

For $z \in M_{F}$ and $f \in C\left(M_{F}\right)$ we define a new process $Y_{\lambda}^{z}=\left\{Y_{\lambda}^{z}(t)\right\}_{t \geq 0}$ with positive parameter $\lambda$ by

$$
Y_{\lambda}^{z}(t)=\frac{1}{\sqrt{\lambda}} \int_{0}^{\lambda t} f\left(X^{z}(s)\right) d s .
$$

Now we are in a position to state the main theorem.

THEOREM 2.2. Let $f$ be a real-valued function in $C\left(M_{F}\right)$ such that there exists $g \in$ $C_{L}^{2}\left(M_{F}\right)$ satisfying $f=\Delta_{L} g$. Consider the process $Y_{\lambda}^{z}$ defined by (13). Then for any quasiregular point $z$ in $Q_{X}$, the processes $Y_{\lambda}^{z}$ converge in law to the Brownian motion $W_{\langle f\rangle}(z)$ with variance $\langle f\rangle(z)$ t as $\lambda \rightarrow \infty$, where $m_{z}$ is the harmonic probability measure associated to the point $z$ and $\langle f\rangle(z)$ is given by

$$
\langle f\rangle(z)=4 \int_{M_{F}}\left(\nabla_{L} g\right)^{2} d m_{z} .
$$


The proof of Theorem 2.2 will be given in Section 4. We finish this section with the following.

REMARK 2.4. (1) For a stationary reversible Markov process, Kipnis and Varadhan [15] proved the same kind of central limit theorem as above under extremely general setting. If we choose a harmonic probability measure $m$ as the initial distribution of the leafwise Brownian motion and consider the process $Y_{\lambda}^{m}=\left\{Y_{\lambda}^{m}(t)\right\}_{t \geq 0}$ instead of $Y_{\lambda}^{z}$, then we can apply their result. But as noted by themselves in Remark 1.7 of [15], their idea is not directly applicable to the limit problem concerned with $m$-almost everywhere initial point $z$. Although there are other papers which prove the central limit theorem with following their approach for a stationary ergodic Markov process (e.g. see [12]), we can not directly apply them by the reason above and the assumption of the ergodicity.

(2) For the sake of convenience we regard the process which is constantly 0 as a Brownian motion with variance 0 in Theorem 2.2 because $\langle f\rangle(z)$ possibly vanishes and the Brownian motion $W_{\langle f\rangle(z)}$ is degenerate. We just give a sufficient condition for non-degeneracy. Let $z \in M_{F}$ be a quasi-regular point. If $f$ satisfies the following conditions (a) and (b), we have $\langle f\rangle(z)>0$, as a consequence the Brownian motion $W_{\langle f\rangle(z)}$ is non-degenerate.

(a) There exists a real-valued element $g \in C_{L}^{2}\left(M_{F}\right)$ such that $f=\Delta_{L} g$. Equivalently, there exist real valued continuous functions $\varphi$ and $\psi$ on $M$ satisfying the equations (4) and (5) for any $x \in M$.

(b) $f$ is not constant on $\operatorname{supp} m_{z}$.

Indeed, from the conditions (b), we can find a point $w \in \operatorname{supp} m_{z}$ such that $f(w) \neq 0$. Therefore from the condition (a) we can find a point $w^{\prime} \in L_{w}$ satisfying $\nabla_{L} g\left(w^{\prime}\right) \neq 0$. Thus $\left(\nabla_{L} g\right)^{2}>0$ holds in a neighborhood $U$ of $w^{\prime}$. On the other hand, from Theorem $2.1, m_{z}$ can be expressed as $m_{\mu}$ for some invariant measure $\mu$ for $F$. Therefore by making use of the Fubini theorem we can conclude that $L_{w} \subset \operatorname{supp} m_{z}$. Hence

$$
(1 / 4)\langle f\rangle(z)=\int_{M_{F}}\left(\nabla_{L} g\right)^{2} d m_{z} \geq \int_{U}\left(\nabla_{L} g\right)^{2} d m_{z}>0 .
$$

Note that the condition (c) below yields the condition (b).

(c) $f$ is not constant along each leaf of $M_{F}$.

It is obvious that if (a) and (c) are satisfied, the variance $\langle f\rangle(z)$ is non-degenerate for any quasi-regular point $z$ for $X$.

(3) Let a harmonic probability measure $m$ for $X$ be given. We give a rather artificial method of constructing a function $f \in C\left(M_{F}\right)$ which satisfies the assumption in Theorem 2.2 and $\langle f\rangle(z)>0$ holds on a set of $m$-measure positive. To this end, we assume that we have real-valued functions $\rho \in C[0,1]$ and $\xi \in C(M)$ such that

(i) The support of $\rho$ is a compact subset of $(0,1)$ and $\int_{0}^{1} u \rho(u) d u \neq 0$.

(ii) Put $\eta=\xi \circ F-\xi$. Then we have $\int_{M}|\eta(x)| d \mu \neq 0$ for an invariant probability measure $\mu$ for $F$ with $m=m_{\mu}$. 
Define a function $\tilde{f}: \mathbf{R} \times M \rightarrow \mathbf{R}$ by $\tilde{f}(u, x)=\rho(u-k) \eta\left(F^{k} x\right)$ for $k \leq u \leq k+1$ and $x \in M$. Then it is obvious that $\tilde{f}$ determines a function $f \in C\left(M_{F}\right)$ with $\tilde{f}(u, x)=$ $f([u, x])$ for $(u, x) \in \mathbf{R} \times M$. We can show that there exists functions $\varphi$ and $\psi$ in $C(M)$ satisfying the equations (4) and (5) in Proposition 2.1. Indeed, if we choose as $\varphi=0$ and $\psi=-\left(\int_{0}^{1} u \rho(u) d u\right) \xi$, then it is easy to see that they satisfies the equations (4) and (5) on $M$. Thus we have verified the condition (a). Next, the condition (ii) yields that $\mu(\eta \neq 0)>0$. Therefore by virtue of Theorem 2.1 , we see that $m_{\mu}\left(Q_{X} \cap \pi_{F}([0,1) \times(\eta \neq 0))\right)>0$. Thus we obtain that $Q_{X} \cap \pi_{F}([0,1) \times(\eta \neq 0))$ is not empty. By virtue of the conditions (i) and (ii), $z \in Q_{X} \cap \pi_{F}([0,1) \times(\eta \neq 0))$ yields that $f$ is not constantly zero on the leaf $L_{z} \subset Q_{X}$. Hence $\langle f\rangle(z)>0$ holds.

(4) In the case of a non-degenerate diffusion with generator $A$ on a compact smooth manifold $M$, it is well-known that for any continuous function $f$ satisfying $\int_{M} f d m=0$, we can find $g \in C^{2}(M)$ such that $A g=f$, where $m$ denotes the unique diffusion invariant probability measure. We shall illustrate later with Example 5.3 that we can not expect this sort of result in the case of the leafwise Brownian motion on a mapping torus in general.

\section{Structure of harmonic measures}

In this section we prove Theorem 2.1. The following elementary inequalities are useful in our arguments.

LEMMA 3.1. Consider the Gaussian kernel $p(t, v)=(1 / \sqrt{2 \pi t}) \exp \left(-v^{2} / 2 t\right)(t>$ 0 , and $v \in \mathbf{R})$. Then we have the following inequalities.

(1) There exists a constant $C_{1}>0$ such that for any $t \geq 1, a \in \mathbf{R}$ and $h \in \mathbf{R}$

$$
\int_{\mathbf{R}}|p(t, v+a+h)-p(t, v+a)| d v \leq \frac{C_{1}|h|}{\sqrt{t}} .
$$

(2) There exists a constant $C_{2}>0$ such that for any $t \geq 1$

$$
\sum_{k \in \mathbf{Z}} \sup _{v \in[k-1, k+1]} p(t, v) \leq 1+\frac{C_{2}}{\sqrt{t}} .
$$

Proof. First of all we note that as a function in $v|(d / d v) p(t, v)|$ is increasing on $(-\infty,-\sqrt{t}) \cup(0, \sqrt{t})$ and decreasing on $(-\sqrt{t}, 0) \cup(\sqrt{t}, \infty)$. It takes maximum $(2 \pi)^{-1 / 2} t^{-1} \exp (-1 / 2)$ at $v= \pm \sqrt{t}$. 
(1) We may assume $h \geq 0$. By the fact we noted above we have

$$
\begin{aligned}
& \sup _{v^{\prime} \in[v+a, v+a+h]}\left|\frac{d p}{d v}\left(t, v^{\prime}\right)\right| \\
& \leq \begin{cases}\frac{1}{\sqrt{2 \pi t}} \frac{-(v+a+h)}{t} \exp \left(\frac{-(v+a+h)^{2}}{2 t}\right) & \text { if } v \leq-(\sqrt{t}+a+h), \\
\frac{1}{\sqrt{2 \pi e}} \frac{1}{t} & \text { if }-(\sqrt{t}+a+h)<v \leq \sqrt{t}-a, \\
\frac{1}{\sqrt{2 \pi t}} \frac{(v+a)}{t} \exp \left(\frac{-(v+a)^{2}}{2 t}\right) & \text { if } v>\sqrt{t}-a .\end{cases}
\end{aligned}
$$

Thus we obtain

$$
\begin{aligned}
\int_{\mathbf{R}}|p(t, v+a+h)-p(t, v+a)| d v \\
\leq h \int_{-\infty}^{-(\sqrt{t}+a+h)} \frac{1}{\sqrt{2 \pi t}} \frac{-(v+a+h)}{t} \exp \left(\frac{-(v+a+h)^{2}}{2 t}\right) d v \\
\quad+h \int_{-(\sqrt{t}+a+h)}^{\sqrt{t}-a} \frac{1}{\sqrt{2 \pi e}} \frac{1}{t} d v \\
\quad+h \int_{\sqrt{t}-a}^{\infty} \frac{1}{\sqrt{2 \pi t}} \frac{(v+a)}{t} \exp \left(\frac{-(v+a)^{2}}{2 t}\right) d v \\
=\frac{1}{\sqrt{2 \pi e}} \frac{h}{\sqrt{t}}+2 \frac{1}{\sqrt{2 \pi e}} \frac{h}{\sqrt{t}}+\frac{1}{\sqrt{2 \pi e}} \frac{h^{2}}{t}+\frac{1}{\sqrt{2 \pi e}} \frac{h}{\sqrt{t}} .
\end{aligned}
$$

Therefore if we further assume $0 \leq h \leq 1$, we obtain the desired inequality with $C_{1}=$ $5 /(\sqrt{2 \pi e})$. For general $h \geq 0$, we write $h=\sum_{j=1}^{k} h_{j}$ with $0 \leq h_{j} \leq 1$. Then we have

$$
\begin{aligned}
& \int_{\mathbf{R}}|p(t, v+a+h)-p(t, v+a)| d v \\
& \quad \leq \sum_{j=1}^{k} \int_{\mathbf{R}}\left|p\left(t, v+a+\sum_{i=1}^{j} h_{i}\right)-p\left(t, v+a+\sum_{i=1}^{j-1} h_{i}\right)\right| d v \\
& \quad \leq \frac{C_{1}}{\sqrt{t}} \sum_{j=1}^{k} h_{j}=\frac{C_{1}|h|}{\sqrt{t}} .
\end{aligned}
$$


(2) For each $k$, we have

$$
\begin{aligned}
& \sup _{v \in[k-1, k+1]} p(t, v) \\
& \leq \inf _{v \in[k-1, k+1]} p(t, v)+\sup _{u, v \in[k-1, k+1]}|p(t, u)-p(t, v)| \\
& \leq \inf _{v \in[k, k+1]} p(t, v)+\sup _{u, v \in[k-1, k]}|p(t, u)-p(t, v)|+\sup _{u, v \in[k, k+1]}|p(t, u)-p(t, v)| \\
& \leq \int_{k}^{k+1} p(t, v) d v+\int_{k-1}^{k} \sup _{v \in[k-1, k]}\left|\frac{d}{d v} p(t, v)\right| d v+\int_{k}^{k+1} \sup _{v \in[k, k+1]}\left|\frac{d}{d v} p(t, v)\right| d v .
\end{aligned}
$$

Therefore we have

$$
\sum_{k \in \mathbf{Z}} \sup _{v \in[k-1, k+1]} p(t, v) \leq \sum_{k \in \mathbf{Z}} \inf _{v \in[k, k+1]} p(t, v)+2 \sum_{k \in \mathbf{Z}} \int_{k}^{k+1} \sup _{v \in[k, k+1]}\left|\frac{d}{d v} p(t, v)\right| d v .
$$

The first term in the right side of (17) is bounded by $\int_{\mathbf{R}} p(t, v) d v=1$. Thus it remains to estimate the second term of the right hand side of (17). Using the fact noted at the beginning of the proof again, it is not hard to see that if $k_{0} \leq \sqrt{t}<k_{0}+1$, we have

$$
\int_{k}^{k+1} \sup _{v \in[k, k+1]}\left|\frac{d}{d v} p(t, v)\right| d v \leq \begin{cases}\int_{k+1}^{k+2}\left|\frac{d}{d v} p(t, v)\right| d v & \text { if } 0 \leq k \leq k_{0}-2, \\ \int_{k-1}^{k}\left|\frac{d}{d v} p(t, v)\right| d v & \text { if } k \geq k_{0}+2 .\end{cases}
$$

In addition, we easily see that

$$
\sum_{k=k_{0}-1}^{k_{0}+1} \int_{k}^{k+1} \sup _{v \in[k, k+1]}\left|\frac{d}{d v} p(t, v)\right| d v \leq \frac{2}{\sqrt{2 \pi}} \frac{1}{t} e^{-1 / 2}+\int_{k_{0}}^{k_{0}+1}\left|\frac{d}{d v} p(t, v)\right| d v
$$

holds. Combining (18) with (19), we obtain

$$
\begin{aligned}
\sum_{k \in \mathbf{Z}} \int_{k}^{k+1} \sup _{v \in[k, k+1]}\left|\frac{d}{d v} p(t, v)\right| d v & =2 \sum_{k=0}^{\infty} \int_{k}^{k+1} \sup _{v \in[k, k+1]}\left|\frac{d}{d v} p(t, v)\right| d v \\
& \leq 2 \int_{1}^{\infty}\left|\frac{d}{d v} p(t, v)\right| d v+\frac{4}{\sqrt{2 \pi}} \frac{1}{t} e^{-1 / 2} \\
& =\frac{2}{\sqrt{2 \pi t}} e^{-1 / 2 t}+\frac{4}{\sqrt{2 \pi}} \frac{1}{t} e^{-1 / 2}
\end{aligned}
$$

Now we have obtained the desired estimate of the second term of the right hand side of (17).

From Lemma 3.1 we have the following. 
LEMMA 3.2. Given any bounded Borel function $f$ on $M_{F}$,

$$
|T(t) f([a, x])-T(t) f([b, x])| \leq \frac{C_{1}\|f\|_{\infty}}{\sqrt{t}}|a-b|
$$

holds for any $a, b \in \mathbf{R}, x \in M$, and $t \geq 1$, where $C_{1}$ is the constant appearing in Lemma 3.1. In particular, if $(1 / t) \int_{0}^{t} T(s) f(z) d s$ converges for some $z \in M_{F}$ as $t \rightarrow \infty$, then $(1 / t) \int_{0}^{t} T(s) f(w) d s$ converges to the same limit for any $w \in L_{z}$ as $t \rightarrow \infty$.

Proof. Assume that $f$ is a bounded Borel function on $M_{F}$. By virtue of the formula (7) and the inequality (15), we have

$$
\begin{aligned}
|T(t) f([a, x])-T(t) f([b, x])| & \leq \sum_{k \in \mathbf{Z}} \int_{[k, k+1)}|f([v, x]) \| p(t, v-a)-p(t, v-b)| d v \\
& \leq\|f\|_{\infty} \int_{\mathbf{R}}|p(t, v-a)-p(t, v-b)| d v \\
& \leq \frac{C_{1}\|f\|_{\infty}}{\sqrt{t}}|a-b| .
\end{aligned}
$$

Next assume that $(1 / t) \int_{0}^{t} T(s) f(z) d s$ converges for some $z=[a, x] \in M_{F}$. Choose any $w=[b, x] \in L_{z}$. Then the inequality just shown in the above implies that

$$
\begin{array}{rl}
\mid \frac{1}{t} \int_{0}^{t} & T(s) f(z) d s-\frac{1}{t} \int_{0}^{t} T(s) f(w) d s \mid \\
& \leq \frac{2}{t}\|f\|_{\infty}+\frac{1}{t} \int_{1}^{t}|T(s) f([a, x])-T(s) f([b, x]) d s| \\
& \leq \frac{2}{t}\|f\|_{\infty}+\frac{C_{1}\|f\|_{\infty}|a-b|}{t} \int_{1}^{t} \frac{1}{\sqrt{s}} d s \\
& \leq \frac{2}{t}\|f\|_{\infty}+\frac{2 C_{1}\|f\|_{\infty}|a-b|(\sqrt{t}-1)}{t} \rightarrow 0 \text { as } t \rightarrow \infty .
\end{array}
$$

Now the proof of the lemma is complete.

Next we give an ergodic theorem for the semi-group.

THEOREM 3.1. Let $m$ be a harmonic probability measure for the leafwise Brownian motion. Then for any $f \in L^{p}(m)$ with $1 \leq p<\infty,(1 / t) \int_{0}^{t} T(s) f(z) d s$ converges $m$ almost everywhere and in $L^{p}(m)$. The limit function $f^{*}$ is an element of $L^{p}(m)$ satisfying $T(t) f^{*}=f^{*}$ in $L^{p}(m)$ and has a version such that for $m$-almost every $z, f^{*}$ is constant on the leaf $L_{z}$.

PROOF. By definition as an operator on $L^{\infty}(m), T(t)$ satisfies $\|T(t)\|_{\infty} \leq 1$ for $t \geq$ 0 . On the other hand, as noted in Remark 2.3, we know that $\|T(t)\|_{1} \leq 1$. Therefore the first assertion follows from the pointwise ergodic theorem Theorem VIII.7.1 and the mean 
ergodic theorem Theorem VIII.7.5 in [6]. It remains to prove the second assertion. For $f \in$ $L^{p}(m)$ with $(1 \leq p<\infty)$, we can find a sequence $f_{n}$ of bounded Borel functions such that $\lim _{n \rightarrow \infty}\left\|f_{n}-f\right\|_{p}=0$. Since $T(t)$ is a contraction on $L^{p}(m)$ for each $t \geq 0$, we have

$$
\left\|\frac{1}{t} \int_{0}^{t} T(s) f_{n} d s-\frac{1}{t} \int_{0}^{t} T(s) f d s\right\|_{p} \leq \frac{1}{t} \int_{0}^{t}\left\|T(s)\left(f_{n}-f\right)\right\|_{p} d s \leq\left\|f_{n}-f\right\|_{p} .
$$

Letting $t \rightarrow \infty$ in the above, we conclude that $\left\|f_{n}^{*}-f^{*}\right\|_{p} \leq\left\|f_{n}-f\right\|_{p}$. Thus we have $\lim _{n \rightarrow \infty}\left\|f_{n}^{*}-f^{*}\right\|_{p}=0$. Taking subsequence if necessary, we may assume $f_{n}^{*}$ converges to $f^{*} m$-a.e. Namely, there exists a Borel measurable set $S_{0}$ with $m\left(S_{0}\right)=1$ such that if $z \in S_{0}, f_{n}^{*}(z)$ converges to $f^{*}(z)$. By virtue of Lemma 3.2 there exists a Borel measurable set $S_{n}$ with $m\left(S_{n}\right)=1$ such that if $z \in S_{n}$, the limit $f_{n}^{*}(w)$ exists for any $w \in L_{z}$ and its value coincides with $f_{n}^{*}(z)$. Put $S=\bigcap_{n=0}^{\infty} S_{n}$. By definition $z \in S$ it is obvious that for any $w \in L_{z}$ $f_{n}^{*}(w)=f_{n}^{*}(z)$ converges to $f^{*}(z)$. This implies that the second assertion is true.

For a while let $\mu$ be an invariant probability measure for the homeomorphism $F$ and $m_{\mu}$ is the measure on $M_{F}$ given by (10). The following corresponds to the fact that the one-dimensional Brownian motion is symmetric with respect to the Lebesgue measure.

Lemma 3.3. Let $f$ and $g$ be bounded Borel measurable functions on $M_{F}$. Then we have

$$
\int_{M_{F}} T(t) f(z) g(z) d m_{\mu}=\int_{M_{F}} f(z) T(t) g(z) d m_{\mu}
$$

for each $t \geq 0$. In particular, the same identity as above holds for $f \in L^{p}\left(m_{\mu}\right)$ and $g \in$ $L^{q}\left(m_{\mu}\right)$, where $1 \leq p, q \leq \infty$ satisfy $1 / p+1 / q=1$ and we regard $1 / q$ as 0 if $q=\infty$.

PROOF. By virtue of (7) we can write the left hand side of the identity of the first assertion as

$$
\begin{array}{rl}
\int_{M_{F}} & T(t) f(z) g(z) d m_{\mu}=\int_{[0,1) \times M} T(t) f([u, x]) g([u, x]) d(l \times \mu) \\
\quad= & \int_{[0,1) \times M}\left(\sum_{k \in \mathbf{Z}} \int_{[0,1)} f\left(\left[v, F^{k} x\right]\right) p(t, v+k-u) d v\right) g([u, x]) d u d \mu .
\end{array}
$$

We note that the boundedness of $f, g$ and the estimate (16) enable us to apply the Fubini theorem repeatedly. The last integral above becomes

$$
\int_{[0,1)} \sum_{k \in \mathbf{Z}}\left\{\int_{M}\left(\int_{[0,1)} f\left(\left[v, F^{k} x\right]\right) p(t, v+k-u) d v\right) g([u, x]) d \mu\right\} d u .
$$


Using $F$-invariance of $\mu$ and substituting $-k$ for $k$, we obtain

$$
\begin{aligned}
\int_{[0,1)} & \sum_{k \in \mathbf{Z}}\left\{\int_{M}\left(\int_{[0,1)} f\left(\left[v, F^{k} x\right]\right) p(t, v+k-u) d v\right) g([u, x]) d \mu\right\} d u \\
= & \int_{[0,1)} \sum_{k \in \mathbf{Z}}\left\{\int_{M}\left(\int_{[0,1)} f([v, x]) p(t, v+k-u) d v\right) g\left(\left[u, F^{-k} x\right]\right) d \mu\right\} d u \\
= & \int_{[0,1)} \sum_{k \in \mathbf{Z}}\left\{\int_{M}\left(\int_{[0,1)} f([v, x]) p(t, v-k-u) d v\right) g\left(\left[u, F^{k} x\right]\right) d \mu\right\} d u \\
= & \int_{[0,1)}\left\{\int_{M}\left(\sum_{k \in \mathbf{Z}} \int_{[0,1)} f([v, x]) p(t, u+k-v) d v g\left(\left[u, F^{k} x\right]\right)\right) d \mu\right\} d u \\
= & \int_{[0,1) \times M}\left(\sum_{k \in \mathbf{Z}} \int_{[0,1)} g\left(\left[u, F^{k} x\right]\right) p(t, u+k-v) d u\right) f([v, x]) d \mu d v \\
= & \int_{[0,1) \times M} f([v, x]) T(t) g([v, x]) d(l \times \mu) \\
= & \int_{M_{F}} f(z) T(t) g(z) d m_{\mu} .
\end{aligned}
$$

Note that we have used the equation $p(t, x)=p(t,-x)$ in the above. Thus the first assertion is verified. The second assertion follows immediately from the fact $T(t)$ is extended to a bounded operator on $L^{p}\left(m_{\mu}\right)$ with $\|T(t)\|_{p} \leq 1$ for each $t \geq 0$.

We summarize the immediate consequences of Lemma 3.3 in Corollary 3.1 below in which the first assertion of Theorem 2.1 is included.

COROLlary 3.1. Let $m_{\mu}$ be the probability measure introduced in the above. Then we have the following.

(1) $m_{\mu}$ is a harmonic measure for the leafwise Brownian motion.

(2) If a nonnegative valued function $h \in L^{1}\left(m_{\mu}\right)$ satisfies $T(t) h=h$ in $L^{1}\left(m_{\mu}\right)$ for any $t \geq 0$, then the measure $h m_{\mu}$ with density $h$ is also a harmonic measure for the leafwise Brownian motion.

Proof. We note that $T(t) 1=1$. Putting $g=1$ in Lemma 3.3, we see that the assertion (1) is valid from Proposition 2.3. Putting $f=h$ in Lemma 3.3, we see that the assertion (2) is valid by the same reason as above.

The rest of this section is devoted to the proof of the second assertion of Theorem 2.1. We take any harmonic probability measure $m$ for the leafwise Brownian motion and fix it. Consider the Borel probability measure $\mu_{m}$ satisfying

$$
\int_{M} \varphi(x) d \mu_{m}=\int_{M_{F}} f_{\varphi}(z) d m
$$


for any $\varphi \in C(M)$, where $f_{\varphi}$ is a function on $M_{F}$ defined so that $f_{\varphi}([u, x])=\varphi(x)$ for each $(u, x) \in[0,1) \times M$. By the Riesz representation theorem such a measure $\mu_{m}$ exists uniquely. We have only to prove the following proposition.

PROPOSITION 3.1. Let $\mu_{m}$ be the probability measure satisfying the identity (20) for any $\varphi \in C(M)$. Then we have $m=m_{\mu_{m}}$.

Proof. We divide the proof into two steps.

(Step 1) $\mu_{m}$ is an invariant measure for $F$.

Let $v$ be any Borel measure on $M_{F}$. Since the projection $\pi_{F}$ restricted to $[0,1) \times M$ is a bimeasurable map onto $M_{F}$, we define a Borel measure $\tilde{v}$ on $[0,1) \times M$ such that

$$
\int_{[0,1) \times M} f([u, x]) d \tilde{v}=\int_{M_{F}} f(z) d v
$$

for any Borel measurable function $f$ on $M_{F}$. For $\varphi \in C(M)$, we have

$$
\begin{aligned}
\int_{M} \varphi(x) d \mu_{m}=\int_{M_{F}} f_{\varphi}(z) d m & =\int_{M_{F}} T(t) f_{\varphi}(z) d m \\
& =\int_{[0,1) \times M} T(t) f_{\varphi}([u, x]) d \tilde{m} \\
& =\int_{[0,1) \times M}\left(\sum_{k \in \mathbf{Z}} \int_{[0,1)} \varphi\left(F^{k} x\right) p(t, v+k-u) d v\right) d \tilde{m}
\end{aligned}
$$

by virtue of (7). Therefore we have

$$
\begin{aligned}
& \left|\int_{M} \varphi(F x) d \mu_{m}-\int_{M} \varphi(x) d \mu_{m}\right| \\
& \quad=\mid \int_{[0,1) \times M}\left(\sum_{k \in \mathbf{Z}} \int_{[0,1)} \varphi\left(F^{k} x\right)(p(t, v+k-1-u)-p(t, v+k-u) d v) d \tilde{m} \mid\right. \\
& \quad \leq \int_{[0,1) \times M}\left(\sum_{k \in \mathbf{Z}} \int_{[0,1)}\left|\varphi\left(F^{k} x\right) \| p(t, v+k-1-u)-p(t, v+k-u)\right| d v\right) d \tilde{m} \\
& \quad \leq\|\varphi\|_{\infty} \int_{\mathbf{R}}|p(t, v-1)-p(t, v)| d v \\
& \quad \leq \frac{C_{1}\|\varphi\|_{\infty}}{\sqrt{t}} \rightarrow 0 \quad(t \rightarrow \infty)
\end{aligned}
$$

from the inequality (15). Hence we reach the desired result.

(Step 2) $m$ is absolutely continuous with respect to $m_{\mu_{m}}$. In fact the density function $h$ satisfies $h \leq 1 m_{\mu_{m}}$-a.e. Since $m$ and $m_{\mu_{m}}$ are probability measures, we conclude that $m=m_{\mu_{m}}$. 
Since $l \times \mu_{m}=\tilde{m}_{\mu_{m}}$, we have only to show that $\tilde{m}$ is absolutely continuous with respect to $l \times \mu_{m}$. To this end it suffices to show that for any bounded nonnegative valued Borel functions $\varphi$ on $M$ and $\psi$ on $[0,1)$

$$
\int_{[0,1) \times M} \varphi(x) \psi(u) d \tilde{m} \leq \int_{[0,1) \times M} \varphi(x) \psi(u) d\left(l \times \mu_{m}\right) .
$$

Let $f_{\varphi, \psi}$ be the function on $M_{F}$ satisfying $f_{\varphi, \psi}([u, x])=\varphi\left(F^{k} x\right) \psi(u-k)$ if $u \in[k, k+1)$. Since $m$ is a harmonic measure, we see from (7) that

$$
\begin{aligned}
\int_{[0,1) \times M} \varphi(x) \psi(u) d \tilde{m}=\int_{M_{F}} f_{\varphi, \psi}(z) d m=\int_{M_{F}} T(t) f_{\varphi, \psi}(z) d m \\
=\int_{[0,1) \times M}\left(\sum_{k \in \mathbf{Z}} \int_{[0,1)} \varphi\left(F^{k} x\right) \psi(v) p(t, v+k-u) d v\right) d \tilde{m} \\
\leq \int_{[0,1)} \varphi(v) d v \int_{[0,1) \times M} \varphi\left(F^{k} x\right) d \tilde{m} \sum_{k \in \mathbf{Z}} \sup _{u \in[k-1, k+1]}\|p(t, u)\|_{\infty}
\end{aligned}
$$

holds for each $t>0$. On the other hand from (20) and Step.1, we obtain

$$
\int_{[0,1) \times M} \varphi\left(F^{k} x\right) d \tilde{m}=\int_{M} \varphi\left(F^{k} x\right) d \mu_{m}=\int_{M} \varphi(x) d \mu_{m} .
$$

Thus the estimate (16) implies that

$$
\int_{[0,1) \times M} \varphi(x) \psi(u) d \tilde{m} \leq\left(1+\frac{C_{2}}{\sqrt{t}}\right) \int_{[0,1) \times M} \varphi(x) \psi(u) d\left(l \times \mu_{m}\right)
$$

for each $t \geq 1$. This yields the inequality (21).

\section{Central limit theorem for additive functionals}

The aim of this section is to prove Theorem 2.2. We make use of the standard arguments which can be found in the proof of the central limit theorem (e.g. Theorem III-4.4 in [13]). Let $z$ be any point in $M_{F}$. We consider the process $Y_{\lambda}^{z}=\left\{Y_{\lambda}^{z}(t)\right\}_{t \geq 0}$ defined by (13), where $f$ is an element in $C\left(M_{F}\right)$ given by $f=\Delta_{L} g$ for a real-valued element $g$ in $C_{L}^{2}\left(M_{F}\right)$. Then we can apply the Itô formula along leaves. Thus we have

$$
g\left(X^{z}(t)\right)-g(z)=\int_{0}^{t} \nabla_{L} g\left(X^{z}(s)\right) d B(s)+\frac{1}{2} \int_{0}^{t} f\left(X^{z}(s)\right) d s .
$$

We denote by $M^{z}(t)$ the martingale term of the right hand side of (22) for the sake of simplicity. Note that the quadratic variation process $\left\langle M^{z}\right\rangle=\left\{\left\langle M^{z}\right\rangle(t)\right\}$ of $M^{z}=\left\{M^{z}(t)\right\}$ is given by

$$
\left\langle M^{z}\right\rangle(t)=\int_{0}^{t}\left(\nabla_{L} g\left(X^{z}(s)\right)\right)^{2} d s .
$$


Then $Y_{\lambda}^{z}(t)$ is given by

$$
Y_{\lambda}^{z}(t)=\frac{2\left(g\left(X^{z}(\lambda t)\right)-g(z)-M^{z}(\lambda t)\right)}{\sqrt{\lambda}} .
$$

In addition we define a family $\left\{M_{\lambda}^{z}\right\}_{\lambda>0}$ of continuous martingales by

$$
M_{\lambda}^{z}(t)=\frac{1}{\sqrt{\lambda}} M^{z}(\lambda t) \quad \text { for } t \geq 0 .
$$

We can easily see that their quadratic variations are given by

$$
\left\langle M_{\lambda}^{z}\right\rangle(t)=\frac{1}{\lambda} \int_{0}^{\lambda t}\left(\nabla_{L} g\left(X^{z}(s)\right)\right)^{2} d s \quad \text { for } t \geq 0 .
$$

We have the following.

LEMMA 4.1. For any $z \in M_{F}, \lambda>0$, and $t, s \in \mathbf{R}$, we have

$$
E\left[\left(M_{\lambda}^{z}(t)-M_{\lambda}^{z}(s)\right)^{4}\right] \leq C_{3}\left\|\nabla_{L} g\right\|_{\infty}^{4}|t-s|^{2}
$$

and

$$
\left|\left\langle M_{\lambda}^{z}\right\rangle(t)-\left\langle M_{\lambda}^{z}\right\rangle(s)\right| \leq\left\|\nabla_{L} g\right\|_{\infty}^{2}|t-s|,
$$

where $C_{3}$ is a positive constant independent of $g, z, \lambda$, $s$ and $t$.

PROOF. From a well-known moment inequality Theorem III-3.1 in [13],we have

$$
E\left[\left(M_{\lambda}^{z}(t)-M_{\lambda}^{z}(s)\right)^{4}\right] \leq C_{3} E\left[\left(\left\langle M_{\lambda}^{z}\right\rangle(t)-\left\langle M_{\lambda}^{z}\right\rangle(s)\right)^{2}\right]
$$

where $C_{3}$ is a universal positive constant. Therefore the first inequality (27) follows easily from the fact the quadratic variation $\left\langle M_{\lambda}^{z}\right\rangle$ is given by (26). The second inequality (28) is also an easy consequence of the equation (26).

We need the following.

LEMMA 4.2. For any $z \in M_{F}$, the family of laws of $C\left([0, \infty) \rightarrow \mathbf{R}^{2}\right)$-valued random variables $\left\{\left(M_{\lambda}^{z},\left\langle M_{\lambda}^{z}\right\rangle\right)\right\}_{\lambda>0}$ is tight. Moreover, if $M_{n}=M_{\lambda_{n}}^{z}$ is any sequence such that the law of $\left(M_{n},\left\langle M_{n}\right\rangle\right)$ converges weakly as $n \rightarrow \infty$, then there exist a probability space $(\tilde{\Omega}, \tilde{\mathcal{F}}, \tilde{P})$ and $C([0, \infty) \rightarrow \mathbf{R})$-valued random variables $\tilde{M}_{n}, \tilde{M}$, and $\tilde{A}$ satisfying the following.

(a) The laws of $\left(M_{n},\left\langle M_{n}\right\rangle\right)$ and $\left(\tilde{M}_{n},\left\langle\tilde{M}_{n}\right\rangle\right)$ coincide for each $n$.

(b) $\lim _{n \rightarrow \infty} \rho\left(\tilde{M}_{n}, \tilde{M}\right)=0$ and $\lim _{n \rightarrow \infty} \rho\left(\left\langle\tilde{M}_{n}\right\rangle, \tilde{A}\right)=0$ hold $\tilde{P}$-a.s.

where $\rho$ is the metric on $C([0, \infty) \rightarrow \mathbf{R})$ given in the proof of Proposition 2.4.

(c) $\tilde{M}$ is a martingale with $\langle\tilde{M}\rangle=\tilde{A}$.

Proof. By the inequality (27) in Lemma 4.1, the family of laws of $\left\{M_{\lambda}^{z}\right\}_{\lambda>0}$ is tight (see Theorem I-4.3 in [13] for example). Since for $C>0$ the subset $\{w \in C([0, \infty) \rightarrow \mathbf{R})$ : $w(0)=0,|w(t)-w(s)| \leq C|t-s|$ for any $t, s \in[0, \infty)\}$ is compact in $C([0, \infty) \rightarrow \mathbf{R})$ 
endowed with the metric $\rho$, the inequality (28) in Lemma 4.1 yields the tightness of the family of laws of $\left\{\left\langle M_{\lambda}^{z}\right\rangle\right\}_{\lambda>0}$. Therefore the family of laws of $C\left([0, \infty) \rightarrow \mathbf{R}^{2}\right.$ )-valued random variables $\left\{\left(M_{\lambda}^{z},\left\langle M_{\lambda}^{z}\right\rangle\right)\right\}_{\lambda>0}$ is tight. Thus by virtue of the Skorohod theorem (Theorem I2.7 in [13]), there exist a probability space $(\tilde{\Omega}, \tilde{\mathcal{F}}, \tilde{P})$ and $C([0, \infty) \rightarrow \mathbf{R})$-valued random variables $\tilde{M}_{n}, \tilde{M}$, and $\tilde{A}$ satisfying the conditions (a) and (b). It remains to show that $\tilde{M}$, and $\tilde{A}$ satisfy the condition (c). To prove the martingale property of $\tilde{M}$, it suffices to show that for any $0 \leq s_{1}<s_{2}<\cdots<s_{k}=s<t$ and bounded continuous function $\varphi: \mathbf{R}^{k} \rightarrow \mathbf{R}$, we have

$$
\tilde{E}\left[\tilde{M}(t) \varphi\left(\tilde{M}\left(s_{1}\right), \ldots, \tilde{M}\left(s_{k}\right)\right)\right]=\tilde{E}\left[\tilde{M}(s) \varphi\left(\tilde{M}\left(s_{1}\right), \ldots, \tilde{M}\left(s_{k}\right)\right)\right] .
$$

Since $\tilde{M}_{n}$ is a martingale, we have

$$
\tilde{E}\left[\tilde{M}_{n}(t) \varphi\left(\tilde{M}_{n}\left(s_{1}\right), \ldots, \tilde{M}_{n}\left(s_{k}\right)\right)\right]=\tilde{E}\left[\tilde{M}_{n}(s) \varphi\left(\tilde{M}_{n}\left(s_{1}\right), \ldots, \tilde{M}_{n}\left(s_{k}\right)\right)\right] .
$$

Clearly, we have $\tilde{M}_{n}(t) \varphi\left(\tilde{M}_{n}\left(s_{1}\right), \ldots, \tilde{M}_{n}\left(s_{k}\right)\right) \rightarrow \tilde{M}(t) \varphi\left(\tilde{M}\left(s_{1}\right), \ldots, \tilde{M}\left(s_{k}\right)\right)$ and $\tilde{M}_{n}(s) \varphi\left(\tilde{M}_{n}\left(s_{1}\right), \ldots, \tilde{M}_{n}\left(s_{k}\right)\right) \rightarrow \tilde{M}(s) \varphi\left(\tilde{M}\left(s_{1}\right), \ldots, \tilde{M}\left(s_{k}\right)\right) \tilde{P}$-a.s. as $n \rightarrow \infty$. In addition, from the inequality (27) in Lemma 4.1, we see that $L^{2}$-norm of integrands in the both side of the equation (30) are uniformly bounded in $n$. Therefore they form sequences of uniformly integrable functions with respect to $\tilde{P}$. Thus each side of the equation (30) converges to each side of the equation (29). Hence the equation (29) is valid.

Since it is obvious that $\tilde{A}$ is an increasing process, it suffices to show that $\tilde{M}^{2}-\tilde{A}$ is a martingale in order to prove $\langle\tilde{M}\rangle=\tilde{A}$. We see that it can be proved in the same way above once we notice that the inequality (27) yields that $L^{2}$-norm of $\tilde{M}_{n}(t)^{2}-\left\langle\tilde{M}_{n}\right\rangle(t)$ is bounded uniformly in $n$ for each $t$. Consequently, $\tilde{M}_{n}(t)^{2}-\left\langle\tilde{M}_{n}\right\rangle(t)$ is uniformly integrable sequence for each $t$.

Now we prove Theorem 2.2.

PROOF OF THEOREM 2.2. Let $f$ and $g$ satisfy the assumptions of the theorem. Obviously we have

$$
\sup _{t \geq 0} \frac{\left|g\left(X^{z}(\lambda t)\right)-g(z)\right|}{\lambda} \leq \frac{2\|g\|_{\infty}}{\lambda} \rightarrow 0 \quad(\lambda \rightarrow \infty) .
$$

Therefore we have only to prove that $\left\{M_{\lambda}^{z}\right\}_{\lambda>0}$ converges in law to the the Brownian motion $(1 / 2) W_{\langle f\rangle(z)}$. The tightness of $\left\{M_{\lambda}^{z}\right\}_{\lambda>0}$ is already established in Lemma 4.2. Thus it remains to identify the limit law under the condition that $z$ is a quasi-regular point for $X$. So in the rest of the proof, we assume that $z$ is a quasi-regular point for $X$. Consider the process $\tilde{M}$ and $\tilde{A}$ obtained in Lemma 4.2. If we can prove that $\tilde{A}(t)=(1 / 4)\langle f\rangle(z) t$ for $t \geq 0$, then we conclude that the law of $\tilde{M}$ coincides with that of $(1 / 2) W_{\langle f\rangle(z)}$ by Theorem II-7.2 in [13]. 
By Proposition 2.4 we see that for each $t>0$

$$
\lim _{\lambda \rightarrow \infty}\left\langle M_{\lambda}^{z}\right\rangle(t)=t \lim _{\lambda \rightarrow \infty} \frac{1}{\lambda t} \int_{0}^{\lambda t}\left(\nabla_{L} g\right)^{2}\left(X^{z}(s)\right) d s=t \int_{M_{F}}\left(\nabla_{L} g\right)^{2} d m_{z}=\frac{1}{4}\langle f\rangle(z) t
$$

holds $P$-a.s. Combining (28) with (32), it is easy to see that $\left\langle M_{\lambda}^{z}\right\rangle(t)$ converges uniformly to $(1 / 4)\langle f\rangle(z) t$ on any compact subset in $[0, \infty) P$-a.s. as $\lambda \rightarrow \infty$. This yields that $\tilde{A}(t)=$ $(1 / 4)\langle f\rangle(z) t$ for $t \geq 0$.

REMARK 4.1. The proof of Theorem 2.2 above is elementary but slightly longwinded. In fact, for a sequence of continuous martingales, its tightness is equivalent to that of the sequence of their quadratic variations. Therefore, the fact that $\left\langle M_{\lambda}^{z}\right\rangle(t)$ converges uniformly to $(1 / 4)\langle f\rangle(z) t$ on any compact subset in $[0, \infty) P$-a.s. as $\lambda \rightarrow \infty$ is sufficient enough to guarantee the validity of the theorem (see [19] and Corollary 1 in [18] for details).

\section{Uniquely ergodic case}

In this section we consider the special case when the underlying homeomorphism $F$ is uniquely ergodic i.e. $\sharp \mathcal{I} \mathcal{M}(F)=1$. The following is an analogue of Theorem 6.19 in [20].

THEOREM 5.1. The following are equivalent.

(1) $F$ is uniquely ergodic.

(2) $\sharp \mathcal{H} \mathcal{M}(X)=1$.

(3) There exists an element $m$ in $\mathcal{H M}(X)$ such that for any $f \in C\left(M_{F}\right)$, $(1 / t) \int_{0}^{t} T(s) f d s$ converges to $\int_{M_{F}} f d m$ uniformly as $t \rightarrow \infty$.

(4) For any $f \in C\left(M_{F}\right)$, there exists a number $C(f)$ depending only on $f$ such that for any $z \in M_{F}$, $(1 / t) \int_{0}^{t} f\left(X^{z}(s)\right) d s$ converges to $C(f)$ in $L^{2}(P)$ as $t \rightarrow \infty$.

(5) For any $f \in C\left(M_{F}\right)$, there exists a number $C(f)$ depending only on $f$ such that for any $z \in M_{F}$, $(1 / t) \int_{0}^{t} f\left(X^{z}(s)\right) d s$ converges to $C(f)$ in $P$ as $t \rightarrow \infty$.

(6) For any $f \in C\left(M_{F}\right)$, there exists a number $C(f)$ depending only on $f$ such that for any $z \in M_{F}$, $(1 / t) \int_{0}^{t} T(s) f(z) d$ s converges to $C(f)$ as $t \rightarrow \infty$.

PROOF. In virtue of Theorem 2.1 the equivalence of the assertions (1) and (2) is obvious.

Next assuming the validity of the assertion (2), we show that of the assertion (3). Suppose that it were not true. Then we could find an element $f_{0}$ in $C\left(M_{F}\right)$, a positive number $\varepsilon_{0}>0$, a sequence $\left\{z_{j}\right\}$ of points in $M_{F}$, and a sequence of positive numbers $\left\{t_{j}\right\}$ such that $\lim _{j \rightarrow \infty} t_{j}=$ $\infty$ and

$$
\left|\frac{1}{t_{j}} \int_{0}^{t_{j}} T(s) f_{0}\left(z_{j}\right) d s-\int_{M_{F}} f_{0} d m\right| \geq \varepsilon_{0} .
$$

Taking a subsequence we may assume that the limit $J(f)=\lim _{j \rightarrow \infty}\left(1 / t_{j}\right) \int_{0}^{t_{j}} T(s) f\left(z_{j}\right) d s$ exists for any $f \in C\left(M_{F}\right)$. By the Riesz representation theorem, there exists a Borel proba- 
bility measure $m^{\prime}$ such that $J(f)=\int_{M_{F}} f d m^{\prime}$ for each $f \in C\left(M_{F}\right)$. By substituting $T(t) f$ for $f$, It is easy to see that $m^{\prime} \in \mathcal{H} \mathcal{M}(X)$. The inequality (33) implies that $m \neq m^{\prime}$. This contradicts the fact that $\sharp \mathcal{H} \mathcal{M}(X)=1$.

Assume assertion (3) is valid. We show (4) with $C(f)=\int_{M_{F}} f d m$. We may assume that $f$ is real-valued. By considering $f-C(f)$, we have only to prove

$$
\lim _{t \rightarrow \infty} E\left[\left(\frac{1}{t} \int_{0}^{t} f\left(X^{z}(s)\right) d s\right)^{2}\right]=0
$$

provided that $(1 / t) \int_{0}^{t} T(s) f d s$ converges to 0 uniformly in $z$ as $t \rightarrow \infty$. For $t>0$ we have

$$
\begin{aligned}
I(t) & =E\left[\left(\frac{1}{t} \int_{0}^{t} f\left(X^{z}(s)\right) d s\right)^{2}\right] \\
& =\frac{2}{t^{2}} \int_{0}^{t} d r \int_{r}^{t} E\left[f\left(X^{z}(r)\right) f\left(X^{z}(s)\right)\right] d s \\
& =\frac{2}{t^{2}} \int_{0}^{t} d r \int_{r}^{t} E\left[f\left(X^{z}(r)\right)(T(s-r) f)\left(X^{z}(r)\right)\right] d s \\
& =\frac{2}{t^{2}} \int_{0}^{t} d r \int_{0}^{t-r} E\left[f\left(X^{z}(r)\right)(T(s) f)\left(X^{z}(r)\right)\right] d s \\
& =\frac{2}{t^{2}} \int_{0}^{t} E\left[f\left(X^{z}(r)\right) \int_{0}^{t-r}(T(s) f)\left(X^{z}(r)\right) d s\right] d r \\
& =\frac{2}{t^{2}} \int_{0}^{t}(t-r) E\left[f\left(X^{z}(r)\right) \frac{1}{t-r} \int_{0}^{t-r}(T(s) f)\left(X^{z}(r)\right) d s\right] d r \\
& =2 \int_{0}^{1}(1-r) E\left[f\left(X^{z}(t r)\right) \frac{1}{t(1-r)} \int_{0}^{t(1-r)}(T(s) f)\left(X^{z}(t r)\right) d s\right] d r .
\end{aligned}
$$

Here the third equality follows from the Markov property of $X$, the fourth equality is obtained by the change of variable $s-r \mapsto s$, the fifth equality is a consequence of the Fubini theorem, and the last equality is obtained by the change of variable $r \mapsto t r$. Thus we have

$$
|I(t)| \leq 2\|f\|_{\infty} \int_{0}^{1}(1-r)\left\|\frac{1}{t(1-r)} \int_{0}^{t(1-r)} T(s) f d s\right\|_{\infty} d r \rightarrow 0 \quad(t \rightarrow \infty)
$$

by the bounded convergence theorem. Hence we have (34) and the assertion (4) is verified.

The assertion (5) follows immediately from the assertion (4) and the assertion (6) is easily verified from the assertion (5). It remains to show that the assertion (6) yields the assertion (2). By Theorem 3.1, we can easily see that $C(f)=\int_{M_{F}} f d m$ holds for any $m \in \mathcal{H M}(X)$. Thus we have $\sharp \mathcal{H} \mathcal{M}(X)=1$. 
We consider a version of Theorem 2.2 for a uniquely ergodic homeomorphism $F$ with $\mathcal{I} \mathcal{M}(F)=\{\mu\}$. Theorem 2.1 implies that $m_{\mu}$ is the unique element in $\mathcal{H} \mathcal{M}(X)$, where $m_{\mu}$ is the measure defined by (10).

THEOREM 5.2. Assume that $M_{F}$ is a mapping torus of a uniquely ergodic homeomorphism $F$. Let $f$ be a real-valued function in $C\left(M_{F}\right)$ which is not constantly 0 on the support of $m_{\mu}$. If there exists a function $g \in C_{L}^{2}\left(M_{F}\right)$ such that $f=\Delta_{L} g$, then we have the following.

(1) The limiting variance is non-degenerate, i.e.

$$
\langle f\rangle=4 \int_{M_{F}}\left(\nabla_{L} g\right)^{2} d m_{\mu}>0 .
$$

(2) Consider the process $Y_{\lambda}^{z}$ defined by (13). Then for any point $z \in M_{F}$, the processes $Y_{\lambda}^{z}$ converge in law to the Brownian motion $W_{\langle f\rangle}$ with variance $\langle f\rangle$ t as $\lambda \rightarrow \infty$.

PROOF. The assertion (1) follows immediately from Remark 2.4 (1).

Now we prove the assertion (2). We use the notation in Theorem 2.2, Lemma 4.1, and Lemma 4.2 in the below. Recall that Lemma 4.1 and Lemma 4.2 are valid whether $z \in M_{F}$ is a quasi-regular point for $X$ or not. Choose any sequence $M_{n}=M_{\lambda_{n}}^{z}$ which converges in law and let $\tilde{M}_{n}$, and $\tilde{A}$ be the processes given in Lemma 4.2 . We have only to show that $\tilde{A}$ satisfies $\tilde{A}(t)=(1 / 4)\langle f\rangle t \tilde{P}$-a.s. for each $t \geq 0$. From (4) of Theorem 5.1, we have

$$
\begin{aligned}
& E\left[\left(\left\langle M_{\lambda}^{z}\right\rangle(t)-\frac{1}{4}\langle f\rangle t\right)^{2}\right] \\
& \quad=E\left[\left(t \frac{1}{\lambda t} \int_{0}^{\lambda t}\left(\nabla_{L} g\right)^{2}\left(X^{z}(s)\right) d s-\int_{M_{F}}\left(\nabla_{L} g\right)^{2} d m_{\mu} \cdot t\right)^{2}\right] \rightarrow 0 \quad(\lambda \rightarrow \infty)
\end{aligned}
$$

for any $z \in M_{F}$ and $t>0$. Since $\left\langle M_{n}\right\rangle$ and $\left\langle\tilde{M}_{n}\right\rangle$ have the same law, we have $\tilde{E}\left[\left(\left\langle\tilde{M}_{n}\right\rangle(t)-\right.\right.$ $\left.(1 / 4)\langle f\rangle t)^{2}\right]=E\left[\left(\left\langle M_{n}\right\rangle(t)-(1 / 4)\langle f\rangle t\right)^{2}\right] \rightarrow 0$ as $(n \rightarrow \infty)$. Thus we arrive at the desired result.

REMARK 5.1. (1) It is well-known that a uniquely ergodic homeomorphism $F$ with $\mathcal{I M}(F)=\{\mu\}$ is minimal if and only if $\mu$ is fully-supported (Theorem 6.17 in [20]). In such a case for any real-valued $g \in C_{L}^{2}\left(M_{F}\right)$ if $f=\Delta_{L} g$ is not constantly 0 , then the limiting variance $\langle f\rangle$ is non-degenerate.

(2) From the proof of Theorem 5.2, we see that the assertion of Theorem 2.2 is valid for the point $z$ such that $(1 / t) \int_{0}^{t}\left(\nabla_{L} g\right)^{2}\left(X^{z}(s)\right) d s$ converges in probability to some constant as $t \rightarrow \infty$. Note that we do not assume the unique ergodicity here. Taking this fact into consideration we introduce another notion of quasi-regularity. We call $z \in M_{F}$ a weakly quasi-regular point for $X$ if for any $f \in C\left(M_{F}\right)$ the $\operatorname{limit}_{\lim } \rightarrow \infty(1 / t) \int_{0}^{t} f\left(X^{z}(s)\right) d s$ exists in probability. We denote by $Q_{X}^{(w)}$ the totality of weakly quasi-regular points for $X$. By 
definition we have $Q_{X} \subset Q_{X}^{(w)}$. It is natural to ask whether they coincide or not. We do not know the answer at present.

We finish with examples.

EXAMPLE 5.1 (generalized Kronecker foliation). Consider the case when $M$ is a compact metrizable group which admits an element $a$ such that the rotation $F: M \rightarrow$ $M, ; x \mapsto a x$ is minimal. As is well-known that the normalized Haar measure $\mu$ is the only invariant probability measure for $F$. When $M$ is the one-dimensional torus and $a=e^{2 \pi \sqrt{-1} \alpha}$ with $\alpha$ being irrational, the corresponding mapping torus is thought of as the so-called Kronecker foliation. So we call the mapping torus $M_{F}$ of $F$ a generalized Kronecker foliation in the sequel.

Let $\hat{M}$ be the character group of $M$. For $n \in \mathbf{Z}$ and $\gamma \in \hat{M}$ we define a function $e_{\gamma, n}$ on $\mathbf{R} \times M$ by $e_{n, \gamma}(u, x)=e^{2 \pi \sqrt{-1}(\alpha(\gamma)+n) u} \gamma(x)$, where $\alpha(\gamma) \in[0,1)$ satisfies $\gamma(a)=$ $e^{2 \pi \sqrt{-1} \alpha(\gamma)}$. Clearly $e_{n, \gamma}(u+1, x)=e_{n, \gamma}(u, a x)$ for any $(u, x) \in \mathbf{R} \times M$. Therefore we regard $e_{n, \gamma}$ as an element in $C_{L}^{\infty}\left(M_{F}\right)$ and write often as $e_{n, \gamma}([u, x])=e_{n, \gamma}(u, x)$. Moreover, it is not hard to verify that the family $\left\{e_{n, \gamma}: \gamma \in \hat{M}, n \in \mathbf{Z}\right\}$ is a complete orthonormal system of $L^{2}\left(m_{\mu}\right)$, where $m_{\mu}$ is the only element in $\mathcal{H} \mathcal{M}(X)$ defined by the equation (10). For $f \in L^{2}\left(m_{\mu}\right)$ its Fourier series expansion is given by

$$
f([u, x])=\sum_{n, \gamma} \hat{f}_{n, \gamma} e_{n, \gamma}([u, x]),
$$

where the summation is taken over all $(n, \gamma) \in \mathbf{Z} \times \hat{M}$ and the Fourier coefficients $\hat{f}_{n, \gamma}$ are given by

$$
\int_{M_{F}} f \overline{e_{n, \gamma}} d m_{\mu}=\int_{[0,1) \times M} f([u, x]) \overline{e_{n, \gamma}(u, x)} d u d \mu
$$

and satisfy $\sum_{n, \gamma}\left|\hat{f}_{n, \gamma}\right|^{2}<\infty$.

We calculate the limiting variance in Theorem 5.2 in terms of the Fourier series. Let $g$ be a real-valued function in $C_{L}^{2}\left(M_{F}\right)$ such that $f=\Delta_{L} g$ is not constantly 0 . Using the expansion (36) we have

$$
\begin{aligned}
g([u, x]) & =\sum_{n, \gamma} \hat{g}_{n, \gamma} e_{n, \gamma}([u, x]), \\
\nabla_{L} g([u, x]) & =\sum_{n, \gamma:(n, \gamma) \neq(0,1)}(2 \pi \sqrt{-1}(\alpha(\gamma)+n)) \hat{g}_{n, \gamma} e_{n, \gamma}([u, x]), \\
f([u, x]) & =\Delta_{L} g([u, x])=\sum_{n, \gamma:(n, \gamma) \neq(0,1)}-4 \pi^{2}(\alpha(\gamma)+n)^{2} \hat{g}_{n, \gamma} e_{n, \gamma}([u, x]) .
\end{aligned}
$$


Thus we have

$$
\begin{aligned}
\langle f\rangle & =4 \int_{M_{F}}\left(\nabla_{L} g\right)^{2} d m_{\mu}=4 \int_{M_{F}}\left|\nabla_{L} g\right|^{2} d m_{\mu} \\
& =16 \sum_{n, \gamma:(n, \gamma) \neq(0,1)} \pi^{2}(\alpha(\gamma)+n)^{2}\left|\hat{g}_{n, \gamma}\right|^{2}>0
\end{aligned}
$$

since $f$ is not constantly 0 .

EXAMPLE 5.2. We consider a special case in Example 5.1 where $M=S^{1}=\{x \in$ C : $|x|=1\}$ and $F x=a x$ is an irrational rotation, i.e. $a=e^{2 \pi \sqrt{-1} \alpha}$ with $\alpha \in(0,1)$ irrational. In this case the mapping torus $M_{F}$ is a foliated manifold which is identified with the so-called Kronecker foliation. The character group $\hat{M}$ is identified with $\mathbf{Z}$ so that if $\gamma \in \mathbf{Z}$, $\gamma(x)=x^{\gamma}$ holds for $x \in M$. Therefore each member $e_{n, \gamma}$ of the above mentioned complete orthonormal system of $L^{2}\left(m_{\mu}\right)$ is given by $e_{n, \gamma}(u, x)=e^{2 \pi \sqrt{-1}(\alpha \gamma+n) u} x^{\gamma}$ and it is not only leafwise smooth but also smooth in the usual sense as a function on the manifold $M_{F}$. Note that we have

$$
T(t) e_{n, \gamma}=e^{-2 \pi^{2}(\alpha \gamma+n)^{2} t} e_{n, \gamma}
$$

for $t \geq 0$. Thus there is no Banach space $(L,\|\cdot\|)$ satisfying conditions (1) and (2) below.

(1) $\left\{e_{n, \gamma}: n \in \mathbf{Z}, \gamma \in \mathbf{Z}\right\} \subset L \subset L^{1}\left(m_{\mu}\right)$.

(2) $T(t)$ is a continuous semi-group on $L$ such that there exists $C>0$ and $\zeta \in(0,1)$ such that

$$
\left\|T(t) f-\int_{M_{F}} f d m_{\mu}\right\| \leq C \zeta^{t}\|f\|
$$

holds for each $f \in L$. Indeed, for any $\varepsilon>0$ we can choose a pair $(n, \gamma) \in \mathbf{Z} \times \mathbf{Z}$ with $\mid \alpha \gamma+$ $n \mid<\varepsilon$ since $\alpha$ is irrational. Thus we are in a different situation from the leafwise Brownian motion on the stable foliation associated with the geodesic flow on the unit tangent bundle over a compact negatively curved manifold. One finds in [17] a Banach space consisting of a class of Hörder continuous functions on which the semi-group of the leafwise Brownian motion satisfies the condition (2).

EXAMPLE 5.3. Let $M_{F}$ be the same mapping torus as in Example 5.2. It is convenient to introduce a real parameter $v$ and denote as $x=e^{2 \pi \sqrt{-1} v}$ in the sequel. Under this convention, $e_{n, \gamma}$ is written as $e_{n, \gamma}(u, v)=e^{2 \pi \sqrt{-1}((\alpha \gamma+n) u+\gamma v)}$.

We give an example of a function $f \in C_{L}^{\infty}\left(M_{F}\right) \cap C^{1}\left(M_{F}\right)$ which satisfies $\int_{M_{F}} f d m_{\mu}=$ 0 but does not have a function $g \in C_{L}^{2}\left(M_{F}\right)$ such that $\Delta_{L} g=f$. To this end, we recall a well-known result in continued fraction (e.g. Theorem 171 in [11]). There exist sequence of positive integers $p_{k}$ and $q_{k}$ such that $p_{k}$ and $q_{k}$ are coprime, $\sum_{k=1}^{\infty}\left(1 / q_{k}\right)<\infty$, and 
$\left|\alpha q_{k}-p_{k}\right|<1 / q_{k}$. Define $f$ by

$$
f(u, v)=\sum_{k=1}^{\infty} \frac{1}{q_{k}^{2}} e_{-p_{k}, q_{k}}(u, v)=\sum_{k=1}^{\infty} \frac{1}{q_{k}^{2}} e^{2 \pi \sqrt{-1}\left(\left(\alpha q_{k}-p_{k}\right) u+q_{k} v\right)} .
$$

Clearly, the right hand side of the above is uniformly convergent in $(u, v) \in \mathbf{R}^{2}$ and $f(u+$ $1, v)=f(u, v+\alpha)$ holds. Therefore $f$ determines a continuous function on $M_{F}$. Since we have $\left(1 / q_{k}\right)^{2}\left|\alpha q_{k}-p_{k}\right|^{r} \leq\left(1 / q_{k}\right)^{r+2}$ for any positive integer $r$, we see that $f$ is of leafwise $C^{r}$ and

$$
\left(\nabla_{L}\right)^{r} f=\sum_{k=1}^{\infty} \frac{\left(2 \pi \sqrt{-1}\left(\alpha q_{k}-p_{k}\right)\right)^{r}}{q_{k}^{2}} e_{-p_{k}, q_{k}} .
$$

On the other hand, $(\partial f / \partial v)(u, v)$ exists and

$$
\frac{\partial f}{\partial v}(u, v)=\sum_{k=1}^{\infty} \frac{2 \pi \sqrt{-1}}{q_{k}} e_{-p_{k}, q_{k}}(u, v)
$$

holds by $\sum_{k=1}^{\infty}\left(1 / q_{k}\right)<\infty$. Therefore, we conclude that $f \in C_{L}^{\infty}\left(M_{F}\right) \cap C^{1}\left(M_{F}\right)$. Finally, we show that there is no function $g \in C_{L}^{2}\left(M_{F}\right)$ satisfying $\Delta_{L} g=f$. If such a function $g$ exists, then its Fourier coefficients $\hat{g}_{n, \gamma}$ with respect to the complete orthonormal system $\left\{e_{n, \gamma}\right\}$ must be given by

$$
\hat{g}_{n, \gamma}= \begin{cases}-\frac{1}{4 \pi^{2} q_{k}^{2}\left(\alpha q_{k}-p_{k}\right)^{2}}, & \text { if } \left.(n, \gamma)=\left(-p_{k}, q_{k}\right) \text { for some } k\right), \\ 0, & \text { otherwise. }\end{cases}
$$

Since we have

$$
\sum_{k=1}^{\infty} \frac{1}{q_{k}^{4}\left|\alpha q_{k}-p_{k}\right|^{4}} \geq \sum_{k=1}^{\infty} 1=\infty,
$$

the norm $\|g\|_{L^{2}\left(m_{\mu}\right)}$ diverges. Hence we arrive at a contradiction.

This example shows that a function $f \in C\left(M_{F}\right)$ with $\int_{M_{F}} f d m_{\mu}=0$ does not always have a leafwise $C^{2}$ solution $g$ of the equation $\Delta_{L} g=f$ even if $f$ has $C^{1}$-regularity in the usual sense.

\section{References}

[ 1] Y. BAKhtin and M. MartíneZ, A characterization of harmonic measures on laminations by hyperbolic Riemann surfaces, Ann. Inst. Henri Poincaré Probab. Stat. 44 (2008), 1078-1089.

[2] A. CAndel, The harmonic measures of Lucy Garnett, Adv. Math. 176 (2003), 187-247.

[3] A. CAndel and L. Conlon, Foliations I, Amer. Math. Soc. Providence, 2000. 
[ 4 ] A. Candel and L. Conlon, Foliations II, Amer. Math. Soc. Providence, 2003.

[ 5 ] M. Denker, C. Grillenberger and K. Sigmund, Ergodic theory on compact spaces, Lecture Notes in Math. 527, Springer-Verlag, Berlin-Hedelberg-New York, 1976.

[ 6] N. Dunford and J. T. Schwartz, Linear Operators Part I, John Wiley \& Sons, Hoboken, 1988.

[ 7 ] R. DURReTt, Stochastic calculus, CRC Press, Boca Raton-London-New York, 1996.

[ 8 ] L. Garnett, Foliations, the ergodic theorem and Brownian motion, J. Funct. Anal. 51 (1983), 285-311.

[9] L. Garnett, Statistical properties of foliations, in "Geometric Dynamics" (J. Palis, Ed.), Lecture Notes in Math. 1007, 294-299, Springer-Verlag, Berlin-New York-Heidelberg, 1983.

[10] U. HAMEnStÄDT, Harmonic measures for compact negatively curved manifolds, Acta Math. 178 (1997), 39-107.

[11] G. H. HARDY and E. M. WRIGHT, An introduction to the theory of numbers, 5th. ed., Oxford Univ. Press, New York, 1979.

[12] H. Holzmann, Martingale approximations for continuous-time and discrete-time stationary Markov processes, Stochastic Process. Appl. 115 (2005), 1518-1529.

[13] N. IKedA and S. WATANABE, Stochastic differential equations and diffusion processes, 2nd. ed., North Holland, Amsterdam, 1989.

[14] V. A. Kaimanovich, Brownian motion on foliations: entropy, invariant measures, mixing, Functional Anal. Appl. 22 (1988), 326-328.

[15] C. Kipnis and S. R. S. Varadhan, Central limit theorem for additive functionals of reversible Markov processes and applications to simple exclusions, Commun. Math. Phys. 104 (1986), 1-19.

[16] F. LEDRAPPIER, Harmonic measures and Bowen-Margulis measures, Israel J. Math. 71 (1990), 275-287.

[17] F. Ledrappier, Central limit theorem in negative curvature, Ann. Probab. 23 (1995), 1219-1233.

[18] R. SH. LiptSER and A. N. SHIRYAEV, On a problem of necessary and sufficient conditions in the functional central limit theorem for local martingales, Z. Wahrsch. 59 (1982), 311-318.

[19] S. NAKAO, On weak convergence of sequences of continuous local martingales, Ann. Inst. Henri Poincaré 22 (1986), 371-380.

[20] P. WALTERs, An introduction to ergodic theory, Springer-Verlag, Berlin-Hedelberg-New York, 1982.

[21] C. YUE, Brownian motion on Anosov foliations and manifolds of negative curvature, J. Diff. Geom. 41 (1995), 159-183.

\author{
Present Addresses: \\ TAKEHIKO MORITA \\ Department of Mathematics, Graduate School of Science, \\ OSAKA UNIVERSITY, \\ TOYONAKA, OSAKA 560-0043, JAPAN. \\ e-mail: take@math.sci.osaka-u.ac.jp \\ KIYOTAKA SUZAKI \\ DePartment of MATHEMATICS, GRAduATE SCHOOL OF SCIENCE, \\ OSAKA UNIVERSITY, \\ TOYONAKA, OSAKA 560-0043, JAPAN. \\ e-mail:k-suzaki@cr.math.sci.osaka-u.ac.jp
}

